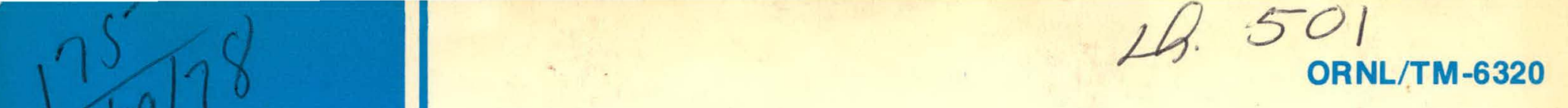

\title{
Status of the Theoretical Study of Microwave Heating in EBT
}

D. B. Batchelor 


\section{DISCLAIMER}

This report was prepared as an account of work sponsored by an agency of the United States Government. Neither the United States Government nor any agency Thereof, nor any of their employees, makes any warranty, express or implied, or assumes any legal liability or responsibility for the accuracy, completeness, or usefulness of any information, apparatus, product, or process disclosed, or represents that its use would not infringe privately owned rights. Reference herein to any specific commercial product, process, or service by trade name, trademark, manufacturer, or otherwise does not necessarily constitute or imply its endorsement, recommendation, or favoring by the United States Government or any agency thereof. The views and opinions of authors expressed herein do not necessarily state or reflect those of the United States Government or any agency thereof. 


\section{DISCLAIMER}

Portions of this document may be illegible in electronic image products. Images are produced from the best available original document. 
Printed in the United States of America. Available from National Technical Information Service

U.S. Department of Commerce

5285 Port Royal Road, Springfield, Virginia 22161

Price: Printed Copy $\$ 5.25$; Microfiche $\$ 3.00$

This report was prepared as an account of work sponsored by an agency of the United States Government. Neither the United States Government nor any agency thereof, nor any of their employees, contractors, subcontractors, or their employees, makes any warranty, express or implied, nor assumes any legal liability or responsibility for any third party's use or the results of such use of any information, apparatus, product or process disclosed in this report, nor represents that its use by such third party would not-infringe privately owned rights. 
ORNL/TM-6320

Dist. Category UC-20 g

Contract No. W-7405-eng-26.

FUSION ENERGY DIVISION

STATUS OF THE THEORETICAL STUDY OF MICROWAVE HEATING IN EBT

D. B. Batchelor

Date Published - September 1978

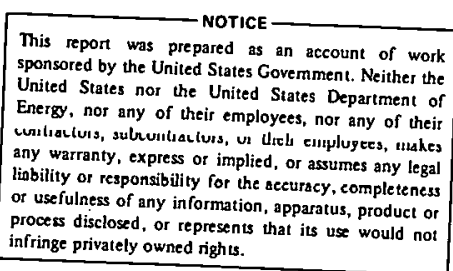

process disclosed, or represents that its use would no
infringe privately owned rights.

Prepared by the

OAK RTDGE NATIONAL LABORATORY

Oak Ridge, Tennessee 37830

operated by

UNION CARBIDE CORPORATION

for the

DEPARTMENT OF ENERGY 
THIS PAGE

WAS INTENTIONALLY

LEFT BLANK 
CONTENTS

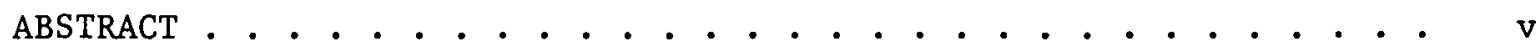

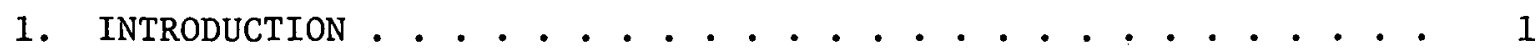

2. MICROWAVE ABSORPTION MECHANISMS • • • • • • • • • • . • . • . • 6

2.1 A Simple Stochastic Heating Model . . . . . . . . . . . . 6

2.2 Wave Damping Model . . . . . . . . . . . . . . . . . 9

3. RAY TRACING IN THREE DIMENSIONS: THE RAYS CODE . . . . . . . . 31

4. JOINING RELATION AT REGIONS OF GEOMETRICAL OPTICS

BREAKDOWN . . . . . . . . . . . . . . . . . . . . . . . 44

5. NONLINEAR PROCESSES • . . . . . . . . . . . . . . . . . . . . . . . . . 49

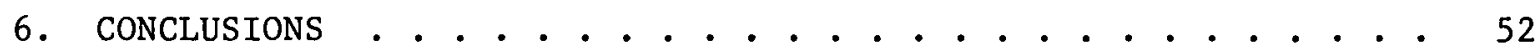

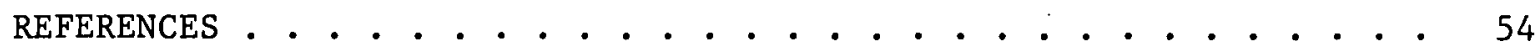




\section{THIS PAGE \\ WAS INTENTIONALLY \\ LEFT BLANK}


The basic strategy of the theoretical study of microwave heating in the ELMO Bumpy Torus (EBT) is outlined and the current status of the various aspects of the study is described. There are four broad areas which are being investigated: (1) heating and wave damping mechanisms, (2) the geometrical optics of microwave propagation in EBT, (3) reflection and mode conversion effects at regions such as cutoff and resonances where the geometrical optics approximation breaks down, and (4) nonlinear effects such as ponderamotive effects and parametric decay. Details are given of the geometrical optics code which has been developed to do ray tracing in arbitrary three dimensional (3-D) plasma equilibria. Examples are given for plasma parameters characteristic of EBT-I and EBT-II. Details are also given of the stochastic heating model currently in use with the 1-D transport code and of the linear wave damping model used in the ray tracing code. The most pressing problems of physics yet to be addressed and the directions for future work are indicated. 
INTRODUCTION

In the EBT devicel all heating is provided by microwaves through the electron cyclotron resonance interaction. In addition, the microwaves play an essential role by producing the high beta, hot electron annuli which provide macroscopic stability for the toroidal plasma component. Clearly, a knowledge of the heating profiles, the microwave field distribution, and the effect of the microwaves on the particle distribution function is crucial to the understanding of EBT operation and scaling. The objective of the EBT microwave heating study is to develop the hasic physics understanding, the calculational techniques, and the computer codes necessary to make detailed practical calculations of the microwave fields and their plasma effects in EBT devices.

Electron cyclotron heating (ECH) is a linear resonance process which has been successfully applied in EBT-I, 1,2 tokamaks, ${ }^{3}$ and other devices. For a review, particularly as applied to EBT-I, see Ref. 2. The basic resonance can be obtained from consideration of electromagnetic waves in a simple, cold, magnetized plasma. For right-hand circularly polarized waves (extraordinary mode) propagating along the magnetic field, the refractive index $n(\omega)$ satisfies the dispersion relation

$\mathrm{n}^{2}(\omega)=\frac{\mathrm{c}^{2} \mathrm{k}^{2}}{\omega^{2}}=1-\frac{\omega_{\mathrm{pe}}^{2}}{\omega\left(\omega-\Omega_{\mathrm{e}}\right)}$,

where

$$
\begin{aligned}
\omega_{p e} & =\text { electron plasma frequency, } \\
\Omega_{e} & =|e| B / m_{e} c=\text { electron cyclotron frequency, and } \\
\omega & =\text { wave frequency. }
\end{aligned}
$$

At a Location in the plasma where $\Omega_{e}=\omega$, the refractive index is infinite and the waves are absorbed. No nonlinear coupling processes are involved, there are no electric field thresholds which must be exceeded, and excessive microwave field intensities are not required. 
A number of previous studies have dealt with the theory of electron cyclotron heating in specific devices. ${ }^{4-11}$ However, these studies have treated the heating due to an arbitrarily specified microwave field structure or have made simplifying assumptions such as plane stratification of the plasma. In EBT, however, the plasma and cavity have very complicated and unsymmetrical geometry (see Fig. 1); thus, the assumption of plane stratification is inadequate, and more detailed calculations are necessary in order to determine the spatial heating profiles.

To solve for the fields directly as a boundary value problem is not practical for several reasons. First, the cavity is large ( $10^{3}$ cubic wavelengths) and discrete cavity modes are not expected to play a significant role. Second, the plasma dielecrr1c properites are nul lucal quantities but depend on the microwave field away from the observation point. Finally, the plasma dielectric properties can be singular (for example, at cutoffs and resonances). However, because the scale lengths for variations in the equilibrium, $\mathrm{L}$, are large in comparison with the wavelengths, $\lambda$, we can use the geometrical optics approximation. That is, we solve the problem by Fourier analyzing in space, expanding in plane waves, and following the local uniform plasma normal modes (ray tracing).

It should be mentioned that for the current device (EBT-I), the conditions for validity of the geometrical optics approximation $(\lambda \ll L)$ are only marginally satistied $(\lambda \sim 1.5-3 \mathrm{~cm}, \mathrm{~L}$ i $3-5 \mathrm{~cm}$ ). Huweves, fur future devices with higher microwave frequencies and larger plasma dimensions, the geometrical optics approximation should be adequate.

Certain difficulties with this approach must be recognized immediately. No matter how gradual the spatlal variations of the plasma, there will be regions where the geometrical optics approximation breaks down. At a cutoff $(k \rightarrow 0)$, the local wavelength becomes infinite and a normai mode of the plasma disappears. At a resonance $(k \rightarrow \infty)$, the local wavelength changes rapidly and a normal wule becvüies singular. If plasma parameters change rapidly, the uniform plasma normal mode description is inappropriate. At any of these regions reflection, absorption, or linear mode conversion (the coupling of two or more normal modes) can occur. Obviously, techniques must be developed to piece together solutions found 


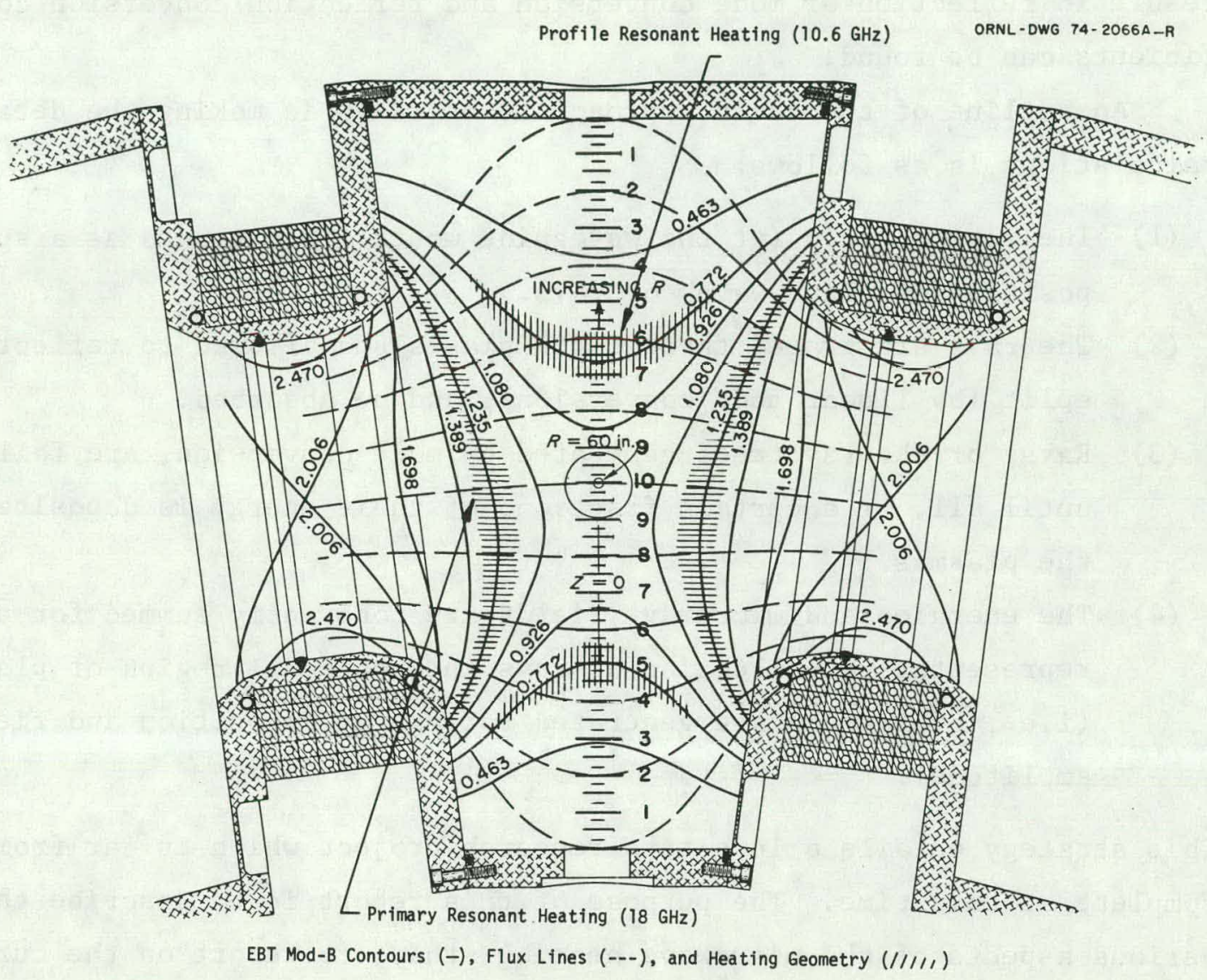

Fig. 1. Configuration of magnetic field in EBT. Two microwave frequencies ( $10.6 \mathrm{GHz}$ and $18 \mathrm{GHz}$ ) are fed from the opening on the inside wall of the cavity. The wave mode at the injection port is ordinary rather than extraordinary. The hot electron ring (runaway electrons) is observed to be a narrow belt encircling the local second harmonic resonance (for $18 \mathrm{GHz}$ ) in the midplane. The maximum microwave heating takes place at the cyclotron resonance zone (hatched area). 
in the regions where geometrical optics is valid across the regions where geometrical optics breaks down. In many cases these singular regions will be thin surfaces only a few wavelengths thick and we can hope to find connection formulas across them. In other cases the singularity will result in reflection or mode conversion and reflection/conversion coefficients can be found.

An outline of the basic approach to be taken in making the detailed calculations is as follows:

(1) The source field (at the waveguide mouth) is expanded as a superposition of plane waves or rays.

(2) The rayo are traced through the plasma and allowed to retlect, split (by linear mode conversion), and be absorbed.

(3) Rays, or the ray trees generated by mode conversion, are followed until all, or a certain fraction, of their energy is deposited in the plasmas.

(4) The energies and microwave fields are coherently summed for a representative collection of rays in each small region of plasma (i.e., histograms are generated for energy deposition and field amplitude).

This strategy entails a long-term research project which is far from complete at this time. The purpose of this report is to describe the various aspects of the microwave heating study, to report on the current status of the research under way, and to indicate the most pressing problems of physics and computer code development yet to be addressed.

Section 2 discusses the microwave absorption mechanisms. Since no nonlinear processes are involved, the basic physics is comparatively well understood. However, absorption calculations in realistic geometries are involved and ultimately coupled to a self-consistent determination of the microwave fields. A simple stochastic heating model currently used in the EBT 1-D transport code is discussed. Techniques are described for calculating the wave damping in a finite temperature plasma, and results are presented for plasma parameters characteristic of EBT-I and for projected EBT-II parameters. In sect. 3, we discuss the geometrical optics code RAYS, which has been developed to do ray tracing in arbitrary $3-D$ plasma 
equilibria. Results are presented for wave propagation in quasi-toroidal equilibria (a straight cylinder with $1 / x$ falloff in magnetic field strength) and for mirror equilibria. In Sect. 4, the problems associated with joining relations at resonances and cutoffs are discussed, and in Sect. 5 some elementary consideration of nonlinear processes is given. Section 6 contains our conclusions. 


\section{MICROWAVE ABSORPTION MECHANISMS}

The resonant absorption of microwave energy by plasma electrons can be understood from several different but ultimately equivalent viewpoints. One approach to microwave heating is to postulate a rf field distribution throughout the plasma and to calculate the encrgy depusition on the basis of single particle orbit theory. A number of papers have used this approach (e.g., Refs. 4, 5, and 6). Of course, the rf fields used are not self-consistent. A very simple, cold plasma model nf thic cort curieuty being used in the EBT 1-D transport code ${ }^{12}$ is described in Sect. 2.1. Another approach is to use the finite temperature dispersion relation to calculate the local value of the imaginary part of the refractive 1ndex for propagating waves. ${ }^{8}, 9,13,14$ This technique, used in the geometrical optics code, is discussed in Sect. 2.2. Finally, local energy absorption can be calculated using the plasma conductivity tensor.7,13,15 This method has computational advantages and is being adopted for use in the RAYS code.

\subsection{A SIMPLE STOCHASTIC HEATING MODEL}

The heating geometry in one section of EBT is shown in Fig. 1. Each t1me an electron passes through a cyclotron resulance zone (hatched in the figure), it gains perpendicular energy from the resonant microwave fleld. Over a time of many transits through the resonant zones, the particle performs a random walk in energy which rcoults in a time-average heating. IL is straightforward to calculate the energy gain per particle on each pass, ${ }^{5}, 11$ and Eldridge has set up a Fokker-P1anck formulaliull which includes the effects of finite temperature, finite wavelength, and relativictic cffecls. ${ }^{10}$ Models of this type are referred to as stochaotir. because an element of randomness is necessary in order to prevent the wave phase and particle gyrophase from becoming correlated on successive passes through the resonant zone. If such correlation develops, the motion becomeo adiabalic, and heating stops when the particles reach a limiting energy. 16 For a device having EBT-I parameters, this adiabatic limit is greater than $30 \mathrm{keV}$ even without considering randomizing effects such as poloidal drift, collisions, or oscillator phase jitter.17 
An electron in an inhomogeneous magnetic field, $\Omega_{e}(z) \cong \omega+\Omega^{\prime} z$, passing through the resonance zone $\Omega_{e}=\omega$ will suffer a change in perpendicular energy $\delta w_{1}$ due to acceleration by the right-circularly rotating component of the electric field $E^{-}=E_{x}-i E_{y}$. This change in energy can be either positive or negative depending upon the gyrophase angle of the electron relative to the wave field. However, when averaged over a random phase angle, the energy gain is positive and proportional to the wave energy density $\left|E^{-}\right|^{2}$ and the length of the time spent in the resonant region $\Delta t=1 / \Omega^{\prime} v_{\|}$,

$<\delta \mathrm{w}_{\perp}>=\frac{\pi \mathrm{e}^{2}\left|\mathrm{E}^{-}\right|^{2}}{2 \mathrm{~m} \Omega^{\prime} \mathrm{v}_{\|}}$

The net heating rate is obtained by multiplying this average energy increment by the electron density and the rate at which electrons pass through the resonant zones, $1 / \tau=v_{\|} / L$,

$\mathrm{P}_{\mu}=\frac{\mathrm{d}}{\mathrm{dt}}\left\langle\delta \mathrm{w}_{\perp}\right\rangle=\left.\frac{\left|\mathrm{E}^{-}\right|^{2}}{2} \frac{\omega_{\mathrm{pe}}^{2}(\mathrm{~s})}{\mathrm{L} \Omega^{\prime}(\mathrm{s})}\right|_{\text {resonant }}$,

where $\mathrm{L}$ is the length of electron excursion along the field lines. Typically $\mathrm{L}$ is taken to be the $\mathrm{z}$ separation between resonant zones in a symmetric mirror.

Note that in this cold plasma, low density model there ls wo interaction between the electrons and the left-circularly polarized (ordinary) mode at cyclotron resonance. The relevant dispersion relation for a leftcircularly polarized wave is

$\mathrm{n}^{2}(\omega)=1-\frac{\omega_{\mathrm{pe}}^{2}}{\omega\left(\omega+\Omega_{\mathrm{e}}\right)}$,

which has no resonances at $\omega=\Omega_{\mathrm{e}}$. 
In order to obtain radial heating profiles from Eq. (3) for use in the EBT 1-D transport code, a bumpy cylinder model for the magnetic field has been adopted:

$\underset{\sim}{B}(\underset{\sim}{x})=B_{1}(z, r) \hat{z}+B_{2}(z, r)\left(\frac{x}{r} \hat{x}+\frac{y}{r} \hat{y}\right)$,

where

$$
\begin{aligned}
B_{1}(z, r) & =\frac{3}{2} B_{0}\left[1-\frac{1}{3} \cos (\kappa z) I_{0}(\kappa r)\right], \\
B_{2}(z, r) & =-\frac{B_{0}}{2} \sin (\kappa z) \bar{I}_{1}(\kappa r), \\
k= & \frac{\pi}{z_{m}}, \\
z_{m} & =\text { axial distance from a mirror midplane to mirror throat, } \\
B_{0} & =\text { field on axis at the midplane, }
\end{aligned}
$$

and $I_{0}(x), I_{1}(x)$ are modified Bessel functinns. This model field bives a mirror ratio of two on axis $(r=0)$. The fields given by Eq. (5) are

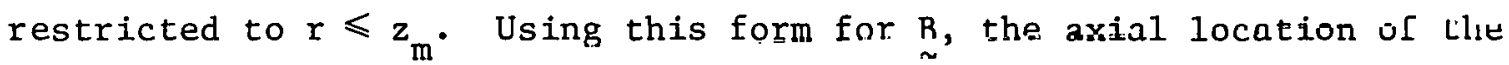
cyclotron resonant zone $z_{R}$ and the gradient of $B$ along a field line can be evaluated analytically as a function of $r$ :

$z_{R}(r)=\cos ^{-1}\left(\frac{3 T_{U}+\left\{\left[4\left(\frac{\omega_{H}}{\Omega_{0}}\right)^{2} \cdot I_{1}^{2}\right]\left(I_{0}^{2}-I_{1}^{2}\right)+9 I_{1}^{2}\right\}^{1 / 2}}{I_{0}^{2}-I_{1}^{2}}\right)$,

$\left.\frac{\partial B}{\partial S}\right|_{\Omega(s)=\omega_{\mu}}=\frac{\kappa B_{0}}{2} \frac{\Omega_{0}^{2}}{\omega_{\mu}^{2}}\left[\sin \left(\kappa z_{R}\right)\left(B_{1}^{2} I_{0}^{2}-B_{2}^{2} I_{1}^{\prime}\right)-2 \cos \left(\kappa z_{R}\right) I_{1} B_{1} B_{2}\right]$, 
where the arguments of $I_{0}, I_{1}$ are $k r$, the quantities $B_{1}$ and $B_{2}$ are evaluated at $\left[z_{R}(r), r\right]$, and $\Omega_{0}=e_{0} / m c$ is the cyclotron frequency on axis at the midplane. In order to model EBT-I, the appropriate parameters are $z_{m}=20 \mathrm{~cm}, B_{0} \cong 5 \mathrm{kG}$, and $\omega_{\mu} / \Omega_{0} \cong 1.3$. Examples of the magnetic field lines and mod-B contours given by this model are shown in Fig. 2 .

It is also necessary to model the microwave electric field profile $\left|E^{-}(r)\right|^{2}$. Typically the 1-D transport calculations have assumed $\left|E^{-}\right|^{2}$ to be constant with $r$. Examples of 1-D transport profiles with this model can be found in Ref. 12. Some calculations have been made with $\left|E^{-}\right|^{2}$ profiles which are depressed in the center as if the microwave energy were depleted by absorption in propagating radially inward. The particular model used was

$\left|E^{-}(r)\right|^{2}=E_{0}^{2}\left[1+\Delta \tanh \left(\frac{r^{2}-r_{0}^{2}}{s^{2}}\right)\right]=E_{0}^{2} H(r)$.

Because no adequate theory exists to determine $E_{0}^{2}$, the microwave field amplitude is fixed by specifying the total power deposited in the toroidal core plasma, $\mathrm{P}_{\text {TOT}}$, which can be estimated from experiment,

$\mathrm{P}_{\text {TOT }}=\int \mathrm{d}^{3} \mathrm{xP}_{\mu}$

Figure 3 shows profiles of power deposited, $P_{\mu}$, in watts per cubic meter, obtained from Eq. (5) for various values $\Delta$ of the on axis depression in $\left|E^{-}(x)\right|^{2}$. The density profile was essentially parabolic.

\subsection{WAVE DAMPING MODEL}

In order to calculate the microwave fields in any way self-consistenlly, it is nccessary to take into account the dielectric properties of the plasma with respect to waves propagating at various angles. The local rate of energy deposition can be determined from the dispersiun relation for waves in a finite temperature plasma. When coupled with ray tracing, in principle this procedure allows the microwave fields to be calculated self-consistently throughout the plasma. 
ORNL/DWG/FED $78-560$

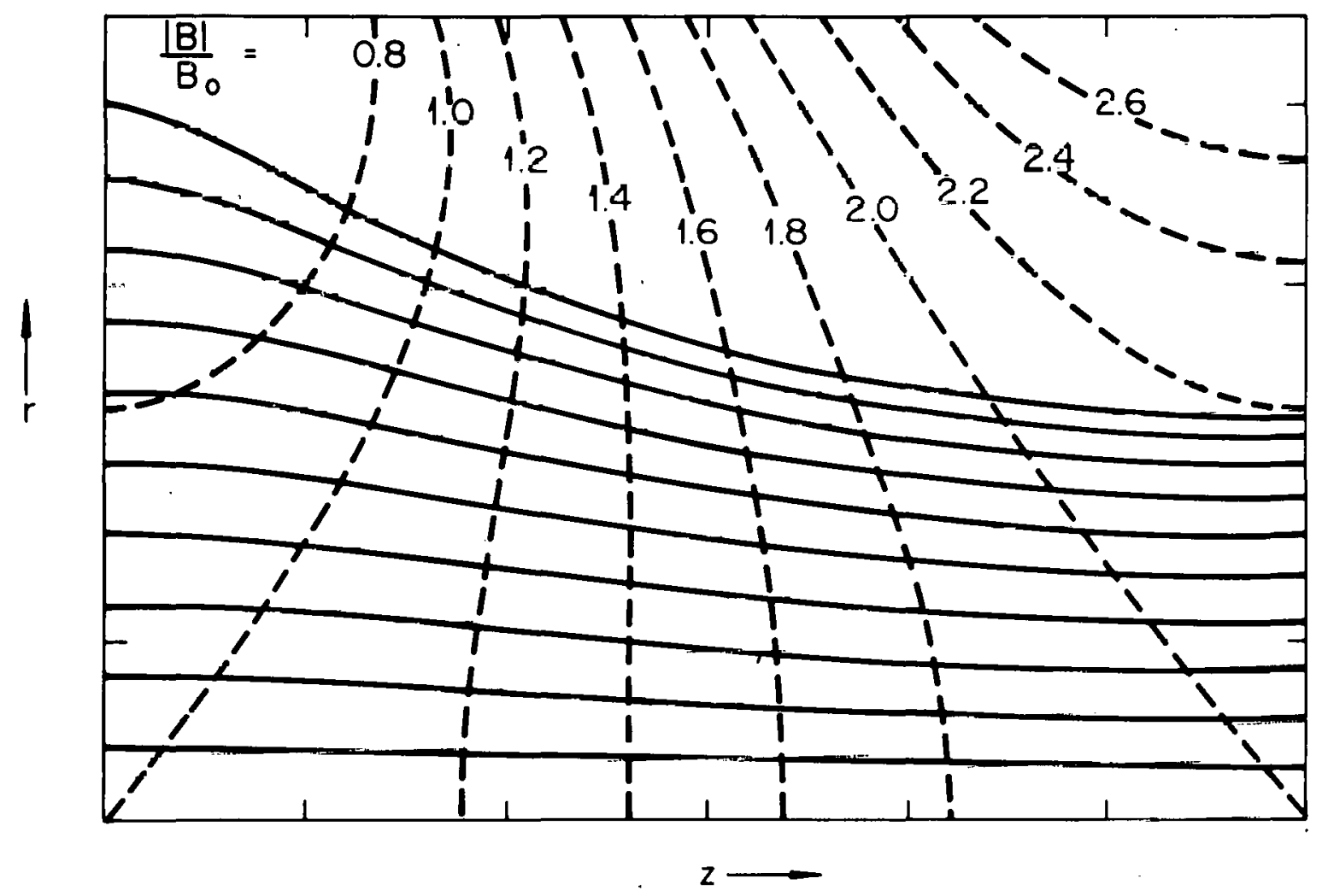

Fig. 2. Field lines and mod-B contours for the model bumpy cylinder magnetic ficld, Fr. (5). 


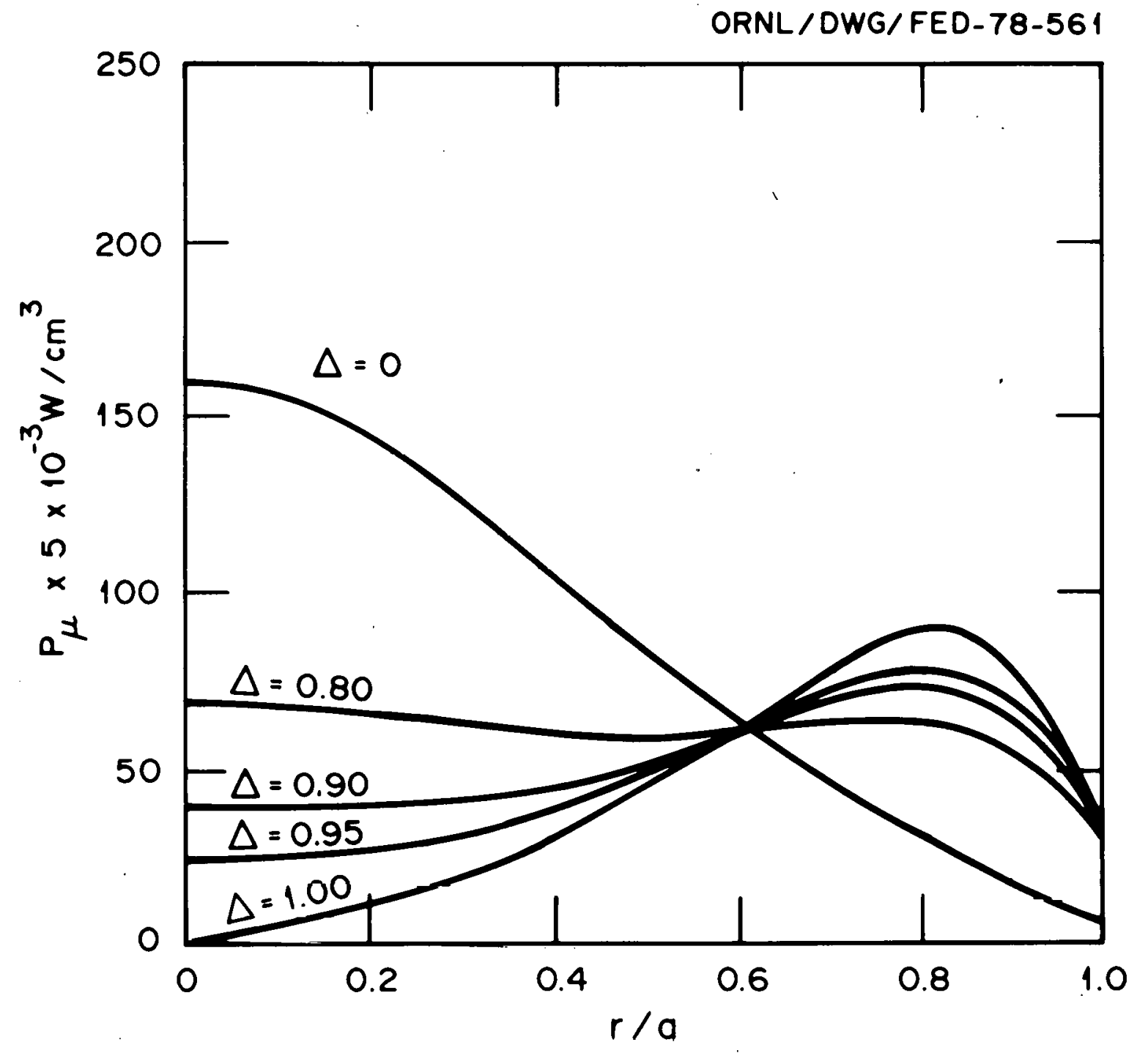

Fig. 3. Radial heating profiles for various shapes of the microwave electric field profile. 
The amount of absorption and the wave propagation characteristics depend sensitively on the plasma geometry as well as on plasma parameters. Considerable simplification is obtained if one assumes that the plasma has a plane stratified geometry (i.e., all gradients in a fixed direction). In that case, the refractive index in a direction perpendicular to the gradients is constant in consequence of Snell's law.

Consider first the case of perpendicular stratification in which the magnetic field is taken along the $\hat{z}$ direction and all variations in plasma parameters are perpendicular to $\mathrm{B}$ in the $\hat{\mathrm{x}}$ direction (Fig. 4). The dispersion relation satisfied by cold plasma waves in such a perpendfeularly stratifled plasma is of the form

$$
\begin{aligned}
& \omega^{4}\left(\omega^{2}-\omega_{\mathrm{pe}}^{2}-\Omega_{\mathrm{e}}^{2}\right) \mathrm{n}_{\mathrm{x}}^{4} \\
& \quad-\omega^{2}\left\{\left(\omega^{2}-\omega_{\mathrm{pe}}^{2}\right)\left(\omega^{2}-\omega_{\mathrm{pe}}^{2} \mathrm{\Omega}_{\mathrm{e}}^{2}\right)+\left(\omega^{2}-\omega_{\mathrm{pe}}^{2}\right)^{2}-\Omega_{\mathrm{e}}^{2} \omega^{2}-\mathrm{n}_{\mathrm{z}}^{2}\left[\left(\omega^{2}-\omega_{\mathrm{pe}}^{2}\right)\left(\omega^{2}-\Omega_{\mathrm{e}}^{2}\right)\right.\right. \\
& \left.\left.+\omega^{2}\left(\omega^{2}-\omega_{\mathrm{pe}}^{2}-\Omega_{\mathrm{e}}^{2}\right)\right]\right\} \mathrm{n}_{\mathrm{x}}^{2} \\
& +\left(\omega^{2}-\omega_{\mathrm{pe}}^{2}\right)\left[\left(\omega^{2}-\omega_{\mathrm{pe}}^{2}\right)^{2}-\omega^{2} \Omega_{\mathrm{e}}^{2}-2 \mathrm{n}_{\mathrm{z}}^{2} \omega^{2}\left(\omega^{2}-\omega_{\mathrm{pe}}^{2}-\Omega_{\mathrm{e}}^{2}\right)+n_{\mathrm{z}}^{4} \omega^{2}\left(\omega^{2}-s_{\mathrm{p}}^{2}\right)\right]=0
\end{aligned}
$$

It can be seen immediately that a resonance $\left(\mathrm{n}_{\mathrm{x}}^{2} \rightarrow \infty\right)$ occurs at the upper hybrid frequency $\omega_{\mathrm{UH}}^{2}=\omega_{\mathrm{pe}}^{2}+\Omega_{\mathrm{e}}^{2}$ for arbitrary values of $\mathrm{n}_{\mathrm{z}}^{2}$. This resonance occurs on the extraordinary mode root of the dispersion relation. cutoffs occur for

$\omega^{2}=\omega_{\mathrm{pe}}^{2}$

and for

$\omega^{2}\left(\omega^{2}-\Omega_{c}^{2}\right) n_{z}^{4}-2 \omega^{2}\left(\omega^{2}-\omega_{\mu e}^{2}-\Omega_{e}^{2}\right) n_{z}^{2}+\left(\omega^{2}-(\omega)_{p e}^{2}\right)^{2}-\Omega_{e}^{2} \omega^{2}=0$.

Equation (11) represents the familiar ordinary mode cutoff. Equation (12) can be solved to yield the magnetic field strength for the extraordinary mode cutoff, 
ORNL / DWG/FEO-78-562

$\underset{\sim}{\underset{\sim}{\nabla}, \underset{\sim}{\nabla} \prod_{e}(x)=\omega}$

$\hat{x}$

$x$

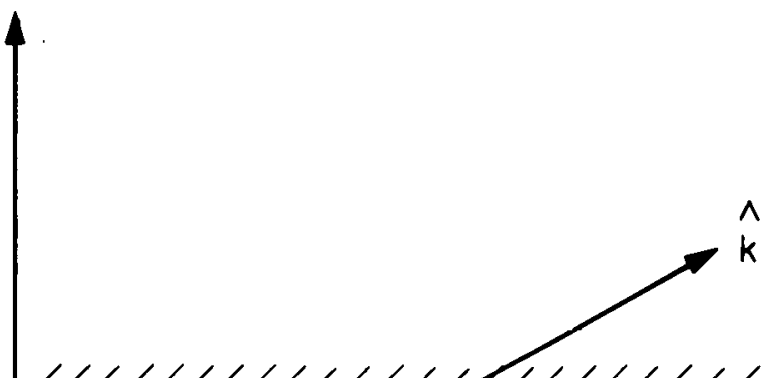

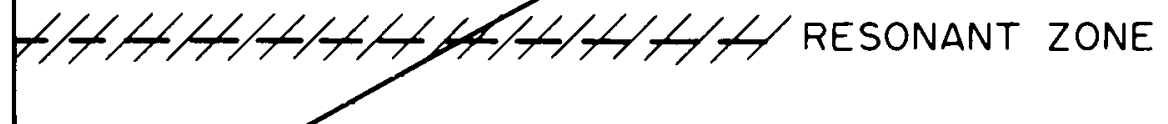

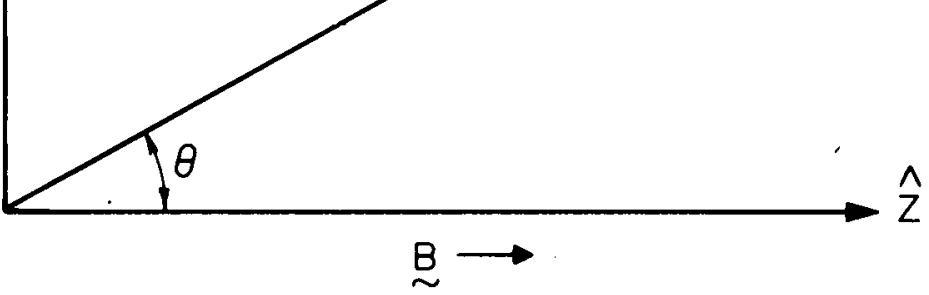

PERPENDICULAR STRATIFICATION

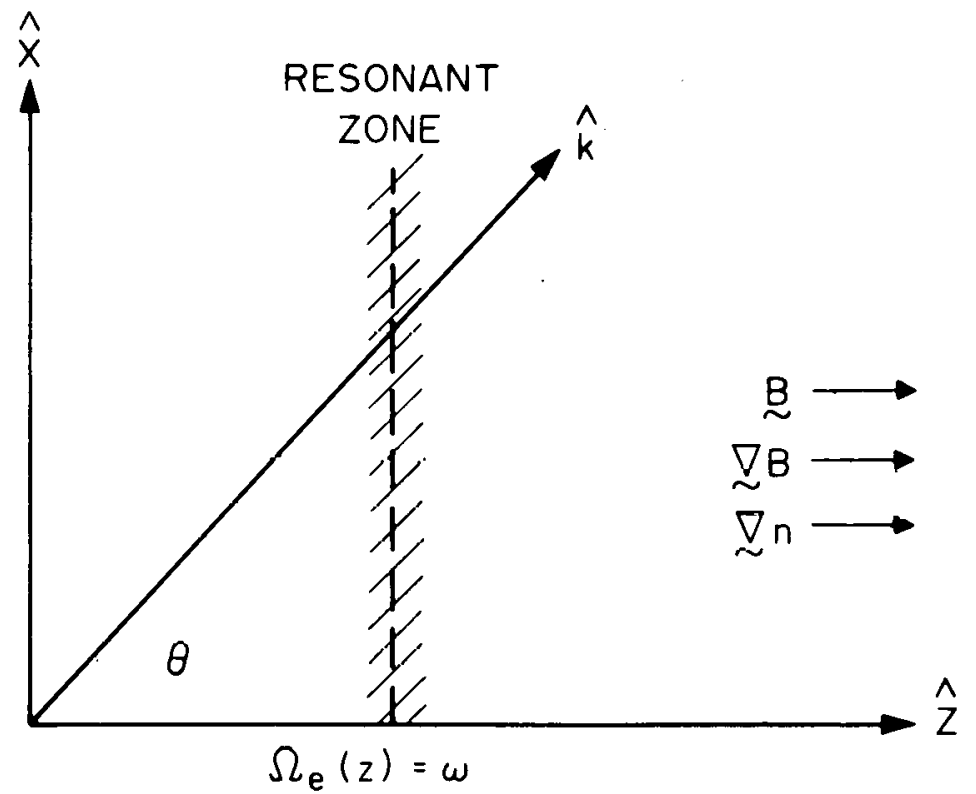

PARALLEL STRATIFICATION

Fig. 4. Geometry for parallel and perpendicularly plane stratified plasmas. 
$\Omega_{e \text { cutoff }}=\omega\left(1-\frac{\omega_{p e^{2} / \omega^{2}}}{1-n_{z}^{2}}\right)$.

Since $\Omega_{\text {e cutoff }}<\omega, E q$. (13) illustrates the well-known result that an extraordinary mode wave propagating in the direction of increasing magnetic field will be reflected when $\Omega_{e}(x)=\Omega_{e}$ cutoff, that is, before reaching the cyclotron resonance layer $\Omega_{e}(x)=\omega$. In the cold plasma model a wave propagating toward the cyclotron resonance from the strong magnetic field side $\left[\Omega_{\mathrm{e}}(\mathrm{x})>\omega\right]$ propagates without impediment past the cyclotron resonance to the upper hybrid. resonance laycr where it must be absorbed or mode converted. As the wave approaches the upper hybrid resonance, $n_{x}$ diverges so the angle between $\underset{\sim}{\mathrm{k}}$ and $\underset{\sim}{\mathrm{B}}$ becomes $\pi / 2$, independent of $\mathrm{n}_{\mathrm{z}}^{2}$. Note that there is no cold plasma resonance at $\Omega_{e}=\omega$ for the case of perpendicular stratification. However, there is a particle resonance at the cyclotron resonant layer, and a finite temperature treatment of the wave propagation shows that energy is weakly absorbed from both ordinary and extraordinary modes. Cyclotron absorption in perpendicularly stratified plasmas has been investigated thoroughly by Eldridge, Namkung, and England 10 for parameters of interest in tnkamaks.

The situation is entirely different for a parallel stratified plasma in which all variations in plasma parametero are in the z direction parallel to $\underset{\sim}{B}$ (Fig. 4). The cold plasma dispersion relation for waves propagating in a parallel stratified medium is of the form

$$
\begin{aligned}
& \omega^{2}\left(\omega^{2}-\omega_{p e}^{2}\right)\left(\omega^{2}-3 l_{e}^{2}\right) n_{z}^{4} \\
& =\omega^{2}\left\{2\left(\omega^{2}-\omega_{p e}^{2}\right)\left(\omega^{2} \omega_{p e^{2}}^{\left.-\Omega_{e}^{2}\right)}-\left[\left(\omega^{2}-\omega_{p e}^{2}\right)\left(\omega^{2}-s e_{e}^{2}\right)+\omega^{2}\left(\omega^{2}-\omega_{p e}^{2}-s l_{e}^{2}\right)\right] n_{x}^{2}\right\} n_{z}^{2}\right. \\
& +\omega^{4}\left(\omega^{2}-\omega_{p e}^{2}-\Omega_{e}^{2}\right) n_{x}^{4}-\omega^{2}\left[\left(\omega^{2}-\omega_{p e}^{2}\right)\left(\omega^{2}-\omega_{p e}^{2}-\Omega_{e}^{2}\right)+\left(\omega^{2}-\omega_{p e}^{2}\right)^{2}-\Omega_{e}^{2} \omega^{2}\right] n_{x}^{2} \\
& +\left(\omega^{2}-\omega_{p e}^{2}\right)\left[\left(\omega^{2}-\omega_{p e}^{2}\right)^{2}-\omega^{2} \Omega_{e}^{2}\right]=0 \text {. }
\end{aligned}
$$


An ordinary mode resonance occurs at $\omega^{2}=\omega_{p e}^{2}$ and an extraordinary mode resonance occurs at $\omega^{2}=\Omega_{e}^{2}$ for arbitrary values of $n_{x}^{2}$. Cutoffs occur at

$\omega_{p e}^{2}=\omega^{2}\left(1-n_{x}^{2}\right)$

for the ordinary mode and at

$\left(\omega^{2}-\omega_{p e}^{2}\right)^{2}-\omega^{2} \Omega_{e}^{2}-\omega^{2}\left(\omega^{2}-\omega_{p e}^{2}-\Omega_{e}^{2}\right) n_{x}^{2}=0$

for the extraordinary mode. Equation (16) can be solved to yleld the magnetic field strength $\Omega_{c}$ for which the extraordinary mode is cut off,

$\Omega_{c}^{2}=\omega^{2}\left(1-\frac{\omega_{p}^{2}}{\omega^{2}}\right)\left(1-\frac{\omega_{p}^{2} / \omega^{2}}{1-n_{x}^{2}}\right)$.

Again for $\omega_{p e} / \omega<1$, we have $\Omega_{c}<\omega$ so that an extraordinary mode wave propagating in the direction of increasing magnetic field will be reflected at the point $\Omega_{e}(z)=\Omega_{c}$ before reaching the cyclotron resonance layer, $\Omega_{e}(z)=\omega$. A wave propagating toward the cyclotron resonance from the high field side reaches the resonance provided the density is such that $\omega_{n \rho}^{2} / \omega^{2}<2-n_{v}^{2}$. Above this density a cutoff exists with $\Omega_{e}$ cutoff $>\omega$. As the wave approaches the cyclotron resonance, $n_{z}$ diverges so the angle between $\underset{\sim}{k}$ and $\underset{\sim}{B}$ approaches zero regardless of the value of $n_{x}^{2}$.

The geometry of EBT is such that the assumption of either parallel or perpendicular stratification is inappropriate, although there are local regions where both approximations can be used. In general, the wave properties and propagation angle near resonance must be obtained by ray tracing. To obtain the rate of energy deposition, one needs the imaginary part of the refractive index for arbitrary angle of propagatiun.

The dispersion relation for electromagnetic waves in a nonrelativistic, uniform, magnetized plasma can be written in the form ${ }^{14}$ 
$\Lambda=\operatorname{det}\left|\Lambda_{i j}\right|=0$,

where

$$
\begin{aligned}
\Lambda_{i j} & =n^{2}\left(\frac{k_{i} k_{j}}{k^{2}}-\delta_{i j}\right)+\varepsilon_{i j}, \\
n & =\frac{c k}{\omega}=\text { refractive index, and } \\
\varepsilon_{i j} & =\text { dielectric tensor. }
\end{aligned}
$$

The elements of the dielectric tensor $\varepsilon_{i j}$ are, in genera1, nomplicated infinite sums of Bessel functions and parallel velocity integra1s. 13,15 The following symmetry relations hold:

$\varepsilon_{21}=-\varepsilon_{12}, \quad \varepsilon_{31}=\varepsilon_{13}, \quad \varepsilon_{32}=-\varepsilon_{23}$.

With these symmetry relations, the determinant in Eq. (1) can be evaluated to give

$\Lambda=\operatorname{det}\left|\Lambda_{i j}\right|=A n^{4}+B n^{2}+C=0$,

where

$$
\begin{aligned}
A= & \varepsilon_{11} \sin ^{2} \theta+2 \varepsilon_{13} \sin \theta \cos \theta+\varepsilon_{33} \cos ^{2} \theta, \\
B= & -\varepsilon_{11} \varepsilon_{33}-\left(\varepsilon_{22} \varepsilon_{33}+\varepsilon_{23}^{2}\right) \cos ^{2} \theta-\left(\varepsilon_{11} \varepsilon_{22}+\varepsilon_{12}^{2}\right) \sin ^{2} \theta \\
& +2\left(\varepsilon_{12} \varepsilon_{23}-\varepsilon_{22} \varepsilon_{13}\right) \sin \theta \cos \theta+\varepsilon_{13}^{2}, \\
C= & \operatorname{det}\left|\varepsilon_{1 j}\right|=\varepsilon_{33}\left(r_{11} \varepsilon_{22}+\varepsilon_{12}^{2}\right)+\varepsilon_{11} c_{23}^{2}+2 \varepsilon_{12} \varepsilon_{13} \varepsilon_{23}-\varepsilon_{22} \varepsilon_{13}^{2} .
\end{aligned}
$$

General expressions for $\varepsilon_{i j}$ when the plasma distribution function is Maxwellian can be found in Refs. 13 and 14 for arbitrary frequency and temperature. However, for $\omega \gg \Omega_{i}=e_{i} B / m_{i} c$, the ion contribution can be neglected. With the additional assumptions 
$k_{1} \rho_{e} \ll 1, \quad \frac{\omega-\ell \Omega_{e}}{k_{1} v_{e}} \gg 1$ for $\quad \ell \neq 1$,

only the $l=0, \pm 1$ terms need to be retained in the sums and the Bessel functions can be expanded to first order in $k_{\perp} \rho_{e}$. The elements of $\varepsilon_{i j}$ then simplify to

$\varepsilon_{11}=\varepsilon_{22}=1-\frac{\omega_{p e}^{2}}{2 \omega\left(\omega+\Omega_{e}\right)}+\frac{\omega_{p e}^{2}}{2 \omega k_{\|} v_{e}} z(\xi)$

$\varepsilon_{12}=-\varepsilon_{21}=-i\left[\frac{\omega_{\mathrm{pe}}^{2}}{2 \omega\left(\omega+\Omega_{e}\right)}+\frac{\omega_{\mathrm{pe}}^{2}}{2 \omega \mathrm{k}_{\|} \mathrm{v}_{\mathrm{e}}} z(\xi)\right]$

$\varepsilon_{13}=\varepsilon_{31}=\frac{\omega_{\mathrm{pe}}^{2}}{\omega \Omega_{\mathrm{e}}} \frac{\mathrm{k}_{1}}{2 \mathrm{k}_{1}}[1+\xi \mathrm{Z}(\xi)]$

$\varepsilon_{23}=-\varepsilon_{32}=i \varepsilon_{13}$

$\varepsilon_{33}=1-\frac{\omega_{\mathrm{pe}}^{2}}{\omega^{2}}+\frac{\omega_{\mathrm{pe}}^{2}}{\omega^{2}} \frac{\omega}{2 k_{4} v_{\mathrm{e}}} \cdot k_{\perp}^{2} \rho_{\mathrm{e}}^{2} \xi[1+\xi Z(\xi)]$

where

$$
\begin{aligned}
Z(\xi) & =\text { plasma dispersiun function, } \\
\xi & =\left(\omega-\Omega_{e}\right) / k_{\|} v_{e}, \\
\rho_{e} & =v_{e} / \Omega_{e} \\
v_{e} & =\sqrt{2^{\prime}{ }_{e} / m_{e}}, \text { and } \\
\Omega_{e} & =|e| B / m_{e} c>0
\end{aligned}
$$

(This notation ditters from Ref. 14, which uses $v_{e}=\sqrt{T_{e} / m_{e}}$ ) 
The plasma dispersion relation $Z(\xi)$ is defined by

$z(\xi)=\frac{1}{\sqrt{\pi}} \int_{-\infty}^{\infty} d x \frac{e^{-x^{2}}}{x-\xi}$,

where the integration is over the Landau contour. In Russian 1iterature, the function $w(\xi)$ is frequently used in place of $Z(\xi)$,

$w(\xi)=\left(1+\frac{2 i}{\sqrt{\pi}} \int_{0}^{\xi} e^{-t^{2}} d t\right) c^{-\xi^{2}}=-\frac{1}{\sqrt{\pi}} z(\xi)$.

To simplify the notation, we introduce the definitions:

$P=\frac{\omega_{p e}^{2}}{\omega^{2}}, \quad q=\frac{\omega_{p e}^{2}}{2 \omega\left(\omega+\Omega_{e}\right)}, \quad \beta=\frac{v_{e}}{c}$,

$\sigma=-\frac{\dot{i}}{2} \frac{\omega_{\mathrm{pe}}^{2}}{\omega \mathrm{k}_{\|} \mathrm{v}_{\mathrm{e}}} \mathrm{Z}(\xi)=\frac{\sqrt{\pi}}{2} \frac{\omega_{\mathrm{pe}}^{2} / \omega^{2}}{\beta \mathrm{n} \cos \theta} \mathrm{w}(\xi)$,

$F=\frac{1}{2} \frac{\mathrm{k}_{\perp}^{2} \rho_{\mathrm{e}}^{2}}{\mathrm{n} \beta \cos \theta} \xi[1+\xi \mathrm{Z}(\xi)]$.

These expressions can now be used in Eq. 22 to obtain

$$
\begin{aligned}
A & =1 \sigma \sin ^{2} \theta+\left\{1-q+P \frac{\omega}{\Omega_{e}}[1+\xi Z(\xi)]\right\} \cdot \sin ^{2} \theta+(1-P+P F) \cos ^{2} \theta, \\
B & =-(1-q+i \sigma)(1-P+P F)\left(1+\cos ^{2} \theta\right)+P^{2} \frac{\omega^{2}}{4 \Omega_{e}^{2}}[1+\xi Z(\xi)]^{2} \tan ^{2} \theta\left(1+\cos ^{2} \theta\right) \\
& -(1-2 q)(1+2 i \sigma) \sin ^{2} \theta-(1-2 q) P \frac{\omega}{\Omega_{e}}[1+\xi Z(\xi)] \tan \theta \sin \theta \cos \theta, \\
C & =(1-2 q)(1+2 i \sigma)(1-P+P F)-(1-2 q) P^{2} \frac{\omega^{2}}{2 \Omega_{e}^{2}}[1+\xi Z(\xi)]^{2} \tan ^{2} \theta .
\end{aligned}
$$


Now compare magnitudes of various terms for $\omega \cong \Omega_{\mathrm{e}}$,

$\sigma \sim \frac{\sqrt{\pi}}{2} \frac{\omega_{\mathrm{pe}}^{2}}{\omega^{2}} \frac{1}{\mathrm{n} \cos \theta} \frac{c}{\mathrm{v}_{\mathrm{e}}} \sim \frac{\omega_{\mathrm{pe}}^{2}}{\Omega_{\mathrm{e}}^{2}} \frac{\mathrm{c}}{\mathrm{v}_{\mathrm{e}}} \frac{1}{\mathrm{n} \cos \theta}$

$F=\frac{1}{2} \frac{\mathrm{k}_{1}^{2} \rho_{\mathrm{e}}^{2}}{\mathrm{n} \beta \cos \theta} \xi[1+\xi Z(\xi)] \sim \mathrm{k}_{\mathrm{e}} \frac{\omega}{\Omega_{\mathrm{e}}} \sin \theta \tan \theta \sim \frac{\mathrm{v}_{\mathrm{e}}}{\mathrm{c}} \frac{\omega^{2}}{\Omega_{\mathrm{e}}^{2}} \mathrm{n} \sin \theta \tan \theta$

$\sim \frac{v_{e}}{c} \tan \theta$

It is generally found that $\omega_{\mathrm{pe}}^{2} / \Omega_{\mathrm{e}}^{2}\left(\mathrm{c} / \mathrm{v}_{\mathrm{e}}\right) \gg 1$, so that $|\sigma| \gg 1$ (this implies a resonably high density plasma if the temperature is high). On the other hand $F$, the $k^{2} \rho_{e}^{2} / n \beta \cos \theta$ term, is small provided $\theta$ is not too near $\pi / 2$. The terms in the dispersion relation, Eq. (20), can be conveniently regrouped,

$\Lambda=i \sigma \Lambda_{0}+\Lambda_{1}+\xi Z(\xi) \Lambda_{2}+\Lambda_{3} F=0$,

where

$$
\begin{aligned}
\Lambda_{0}= & \sin ^{2} \theta n^{4}-\left[(1-P)\left(1+\cos ^{2} \theta\right)+(2-4 q) \sin ^{2} \theta\right] n^{2} \\
& +2(1-P)(1-2 q) \\
\Lambda_{1}= & {\left[\left(1-q+P \frac{\omega}{\Omega_{e}}\right) \sin ^{2} \theta+(1-P) \cos ^{2} \theta\right] n^{4} } \\
& -\left[(1-q)(1-P)\left(1+\cos ^{2} \theta\right)-P^{2} \frac{\omega^{2}}{4 \Omega_{e}^{2}}\left(1+\cos ^{2} \theta\right) \tan ^{2} \theta\right.
\end{aligned}
$$




$$
\begin{aligned}
& \left.+\left(1+p \frac{\omega}{\Omega_{e}}\right)(1-2 q) \sin ^{2} \theta\right] n^{2} \\
& +(1-P)(1-2 q)-P^{2} \frac{\omega^{2}}{2 \Omega_{e}^{2}}(1-2 q) \tan ^{2} \theta \\
& \Lambda_{2}=P \frac{\omega}{\Omega_{e}} \sin ^{2} \theta n^{4}-\left[(1-2 q) P \frac{\omega}{\Omega_{e}} \sin ^{2} \theta-\frac{P}{4}-\frac{\omega_{p e}^{2}}{\Omega_{e}^{2}} \tan ^{2} \theta\left(1+\cos ^{2} \theta\right)\right] n^{2} \\
& -\frac{P}{2}(1-2 q) \frac{\omega_{p e}^{2}}{\Omega_{e}^{2}} \tan ^{2} \theta \\
& \Lambda_{3}=P \cos ^{2} \theta n^{4}-(1-q) F\left(1+\cos ^{2} \theta\right) n^{2}+r(1-2 q) .
\end{aligned}
$$

Equation (26) describes the propagation and damping of electromagnetic waves propagating at arbitrary angles in a nonrelativistic plasma of arbitrary density and temperature. The only approximations made were $k_{1} \rho_{e} \ll 1$ and $\left(\omega-\ell \Omega_{e}\right) / k_{\|} v_{e} \gg 1$ for $\ell \neq 1$. The nonrelativistic formulation breaks down, however, for angles of propagation very near $\theta=\pi / 2$. In the case that $n_{\|} \leqslant v_{e} / c$, broadening of the resonance $\left(\omega-k_{\|} v_{\|}-\right.$ $\ell \Omega / \gamma)^{-1}$ due to the relativistic mass increase, $\gamma$, is comparable to the Doppler broadening, $k_{\|} v_{\|}$, and must be included.

Calculations based on a weak damping approximation to the dispersion relation, Eq. (20), date from the mid-1950's and are well known. Such an approximation has been used in the RAYS code to calculate the absorption ot energy along a ray in varioue plaoma equilibid. Slnce a number of misprints exist in the published literature, and the form of the equaliums used in the RAYS code is slightly more general, the results of the weak dämping analysis are reproduced here.

Using the assumption that

$\frac{\omega_{\mathrm{pe}}^{2}}{\omega^{2}} \frac{\mathrm{c}}{\mathrm{nv}_{\mathrm{e}} \cos \theta} \gg 1$, 
we find that $|\sigma| \gg 1$ so the first term in Eq. (20) is dominant. Then to lowest order the refractive is given by $\mathrm{n}_{ \pm}^{2}$ where

$$
\begin{aligned}
& \Lambda_{0}\left(\mathrm{n}_{ \pm}^{2}\right)=0 \text { and } \\
& \begin{aligned}
\mathrm{n}_{ \pm}^{2}= & \left(2-\mathrm{P}\left(1+\cos ^{2} \theta\right)+(1-4 q) \sin ^{2} \theta \pm\left\{\mid 2-P\left(1+\cos ^{2} \theta\right)\right.\right. \\
& \left.\left.\left.\quad+(1-4 q) \sin ^{2} \theta\right]^{2}-8(1-P)(1-2 q) \sin ^{2} \theta\right\} 1 / 2\right) / 2 \sin ^{2} \theta
\end{aligned}
\end{aligned}
$$

When evaluated at $\omega=\Omega_{\mathrm{e}}$ these refractive indices agree with cold plasma results with the positive sign corresponding to extraordinary mode and the negative to ordinary. The first order correction to the refractive index is obtained by expanding the dispersion relation around $n_{ \pm}^{2}$,

$\Lambda(n) \cong \Lambda\left(n_{ \pm}\right)+\left(n-n_{ \pm}\right) \frac{\partial \Lambda}{\partial n_{ \pm}}$

Using Eq. (20) gives

$\delta n=n-n_{ \pm}=i \frac{\Lambda_{1}\left(n_{ \pm}^{2}\right)+\Lambda_{2}\left(n_{ \pm}^{2}\right) f\left(n_{ \pm}^{2}\right)+\Lambda_{3}\left(n_{ \pm}^{2}\right) F\left(n_{ \pm}^{2}\right)}{\sigma\left(n_{ \pm}\right) \partial \Lambda_{0} / \partial n_{ \pm}}$.

The term $\Lambda_{3} F$ is small by a factor $v_{e} / c$ and can be neglected. The $\Lambda_{2} f$ term makes a first order correction to the real part of the refractive index, and the $\Lambda_{1}$ term givcs the imaginary part of the refractive index,

$$
\mathrm{n}_{i} \bar{\tau}-\frac{2 v_{e} / c \cos \theta n_{ \pm} \Lambda_{1}}{\frac{\omega_{p e}^{2}}{\omega^{2}} \frac{\partial \Lambda_{0}}{\partial n_{ \pm}}} \operatorname{Im}\left[\frac{1}{z(\xi)}\right] .
$$

A number of comments should be made concerning the validity of this approximation. The approximation $|\sigma| \gg 1$ can break down if $\omega$ is too 
close to cold plasma resonance since $\mathrm{n}^{2}$ becomes large there. In particular, for the extraordinary mode a cold plasma resonance occurs for $\Omega_{e}$ such that

$\frac{\Omega_{e}^{2} \text { resonant }}{\omega^{2}}=\frac{1-\frac{\omega_{\text {pe }}^{2}}{\omega^{2}}}{1-\frac{\omega_{\text {pe }}^{2}}{\omega^{2}} \cos ^{2} \theta}$.

f'ur angles of propagation near $\theta=\pi / 2$ the cold plasill resonance occurs neãr the upper hybrid frequency $\Omega_{U H}=\left(\Omega_{e}^{2}+\omega_{p e}^{2}\right)^{1 / 2}$, the plasma resonance and cyclotron resonance are well separated, $\mathrm{n} \cos \theta$ is small, and the inequality (27) is easily satisfied for $\left(\omega_{\mathrm{pe}}^{2} / \omega^{2}\right) c / v_{e} / \omega^{2} v_{e}>1$. We therefore expect the weak damping approximation always to be valid for the extraurdinary mode when the ray approaches the cyclotron resonance with $\underset{\sim}{k}$ nearly perpendicular to $\underset{\sim}{\mathrm{B}}$. This is the case, for example, in a perpendicularly stratified medium, Eq. (10).

For angles of propagation near $\theta=0$, the cold plasma resonance occurs near the cyclotron frequency, $n_{\|}$becomes very large, and inequality (27) is violated for $\Omega_{c} \cong \omega$. For $\theta=0$ even in the limit $v_{c} / c \rightarrow 0$, the . contribution of $\Lambda_{1}$ in Eq. (26) must be retained to obtain the cold plasma dispersion relation. For example, in a perpendicularly stratified plasma the angle of propagation $\theta$ vanishes as the cyclotron layer is approached, $\mathrm{n}_{z}$ diverges, and the weak damping approximation will always break down for $\Omega$ sufficiently near 11 . In this caoc both the real and imaginary parts of $\mathrm{n}$ become large at the cyclotron resonance and the wave is completely absorbed.

Fur the ordinary mode, no cold plasma resonances occur near $\Omega_{e}=\omega$ so that cyclotron absorption is a comparatively weak finite-temperature effect. Accordingly, the weak damping approximation is valid for any plasma geometry and for almost all angles of propagation. However, for angles very near $\dot{\theta}=\pi / 2$, the relativistic correction is required and the approximation can break down due to the presence of $\tan ^{2} \theta$ in terms in $\Lambda_{1}$. 
Finally, it should be mentioned that if the quantities $\Lambda_{1}, \Lambda_{2}$, and $\partial \Lambda_{0} / \partial n_{ \pm}$are evaluated at $\Omega_{e}=\omega$ then $\mathrm{Eq}$. (31) reduces to the results contained in Alhiezer et al. (Ref. 14)(their expression for $\Lambda_{1}$ contains a misprint, however). For small values of $v_{e} / c$ and angles near $\theta=\pi / 2$ the resonance is quite narrow, and since the $\Lambda$ 's are slowly varying functions of $w$ they can be evaluated at $\Omega_{e}=\omega$. However, for larger values of $v_{e} / c$ and small angles the resonance is broad and the $\Lambda$ 's do vary somewhat across the resonance. Better agreement is obtained on the wings of the absorption line between the weak damping approximation and an exact solution of the dispersion if the $\Lambda$ 's are evaluated at the local value of $\Omega_{e}$.

Figure 5 shows the imaginary part of $\underset{\sim}{\mathrm{k}}$ in (centimeters) ( $^{-1}$ vs $\Omega_{\mathrm{e}} / \omega$ for plasma parameters typical of EBT-I: $\omega / 2 \pi=18 \mathrm{GHz}, \mathrm{T}_{\mathrm{e}}=300 \mathrm{eV}\left(\mathrm{v}_{\mathrm{e}} / \mathrm{c}=\right.$ $0.034)$, and $\omega_{\mathrm{pe}}^{2} / \omega^{2}=0.3-0.7\left(\mathrm{n}_{0}=1.2-2.8 \times 10^{12} / \mathrm{cm}^{3}\right)$. Figures $5(\mathrm{a})$ and 5 (b) are for ordinary and extraordinary modes, respectively, at a propagation angle $\theta=85^{\circ}$, while Figs. $5(\mathrm{c})$ and $5(\mathrm{~d})$ are for ordinary and extraordinary modes at $\theta=45^{\circ}$. Figure 6 shows the imaginary part of $\underset{\sim}{\mathrm{k}}$ in (centimeters) ${ }^{-1}$ vs $\Omega_{\mathrm{e}} / \omega$ for typical projected plasma parameters for EBT-II: $\omega / 2 \pi=120 \mathrm{GHz}, \mathrm{T}_{\mathrm{e}}=2 \mathrm{keV}\left(\mathrm{v}_{\mathrm{e}} / \mathrm{c}=0.09\right)$, and $\omega_{\mathrm{pe}}^{2} / \omega^{2}=0.3\left(\mathrm{n}_{0}=\right.$ $\left.5.36 \times 10^{13}\right)$ to $\omega_{\mathrm{pe}}^{2} / \omega^{2}=0.7\left(\mathrm{n}_{0}=1.25 \times 10^{14} / \mathrm{cm}^{3}\right)$.

A quantity of considerable interest is the fraction $f$ of the power absorbed from a wave as it propagates through the cyclotron resonance. This can be expressed in terms of a total damping coefficient $\alpha_{T}$

$f=1-e^{-\alpha} T$

where

$$
\begin{aligned}
\alpha_{T} & =\int_{-\infty}^{\infty} \mathrm{d} s 2 \mathrm{k}_{i}(s) \cos [\phi(s)]=4 \pi \frac{\omega}{c} \int_{-\infty}^{\infty} \mathrm{ds} \mathrm{n}_{i}(s) \cos [\phi(s)], \\
s & =\operatorname{arc} \text { length along a ray, and } \\
\phi(s) & =\text { angle between } \underset{\sim}{\mathrm{k}} \text { and the group velocity } \underset{\sim}{\mathrm{v}}=\partial \omega / \partial \underset{\sim}{\mathrm{k}} .
\end{aligned}
$$



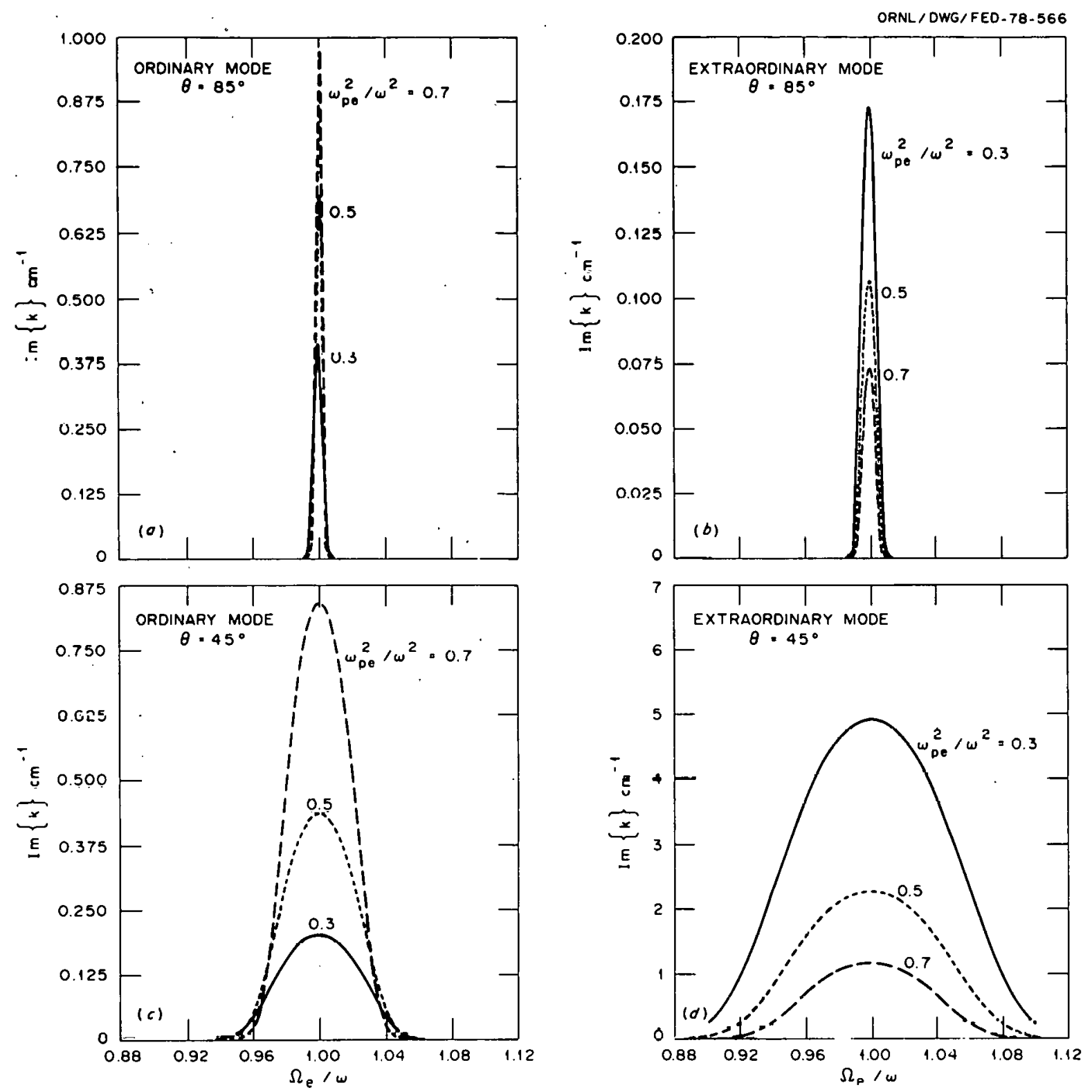

Fig. 5. Imaginary part of $k$ in (centimeters) ${ }^{-1}$ for ordinary and extraordinary modes with $\theta=85^{\circ}$ and $45^{\circ}$ for various values of $\omega_{\mathrm{pe}}^{2} / \omega^{2}$. The parameters are characteristic of EBT-I: $\omega / 2 \pi=18 \mathrm{GHz}, \mathrm{v}_{\mathrm{e}} / \mathrm{c}=0.034$. 

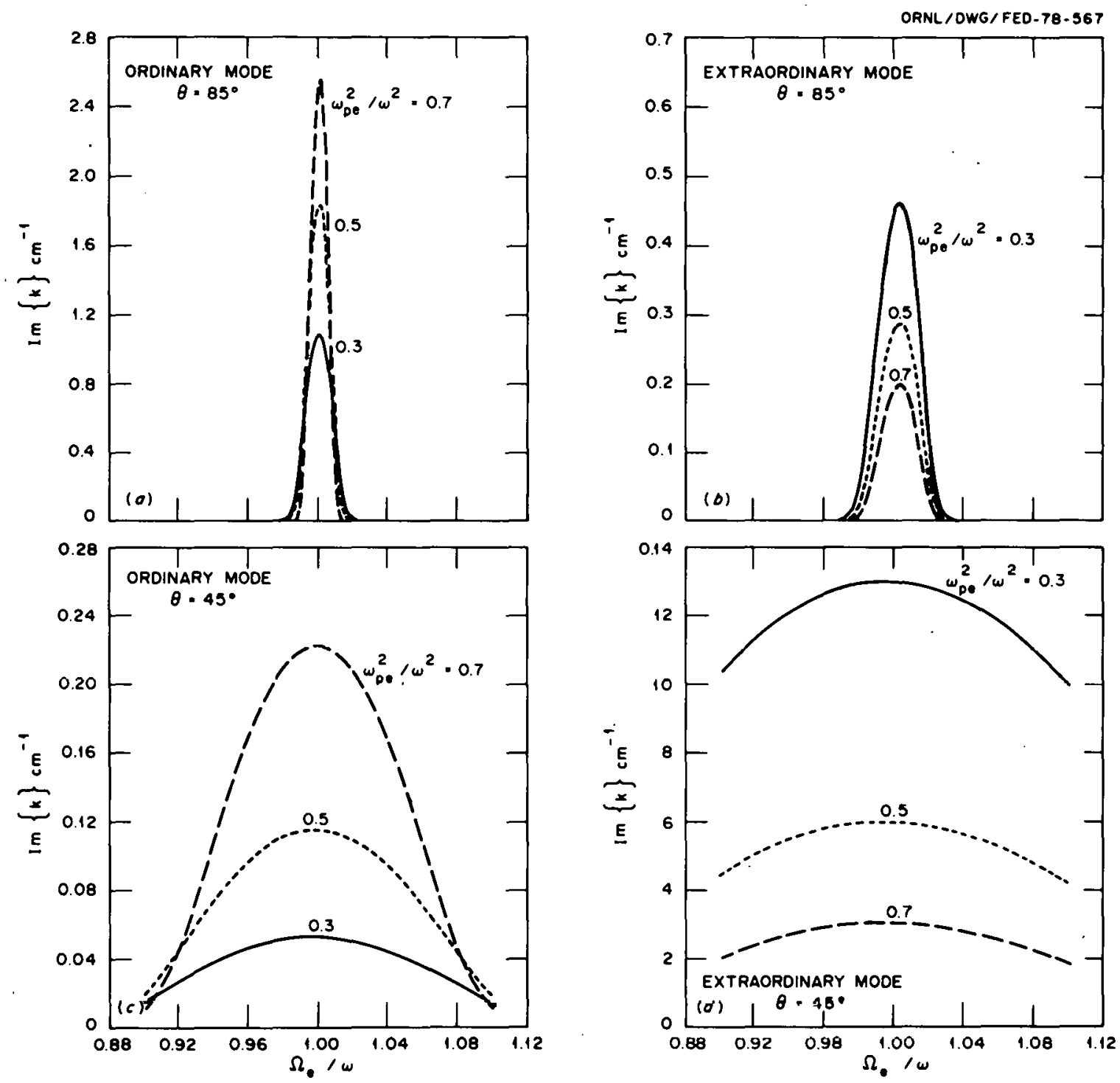

Fig. 6. Imaginary part of $\mathrm{k}$ in (centimeters) - $^{-1}$ for ordinary and extraordinary modes with $\theta=85^{\circ}$ and $45^{\circ}$ for various values of $\omega_{\mathrm{pe}}^{2} / \omega^{2}$. The parameters are characteristic of projections for EBT-II: $\omega / 2 \pi=$ $.120 \mathrm{GHz}, \mathrm{v}_{\mathrm{e}} / \mathrm{c}=0.09$. 
This integral is calculated numerically in the RAYS code to determine the power deposited along a ray. However, if the ray remains sufficiently far from a cold plasma resonance (e.g., ordinary mode, or extraordinary mode in a perpendicularly stratified medium), then $\underset{\sim}{\mathrm{k}}$ is nearly parallel to $\underset{\sim \mathrm{g}}{\mathrm{v}}$ and the real part of $\mathrm{k}$ changes very little through the cyclotron resonance layer. In that case the integral can be done analytically to yield estimates of the fractional damping. Making use of Eq. (31) and evaluating $\mathrm{n}_{ \pm}$and the $\Lambda^{\prime}$ 's at $\Omega_{e}=\omega$, one finds that only the argument $\xi$ of the $z$ function varies with $s, \xi=(1-\Omega(s) / \omega) /\left(n_{ \pm} \cos \theta v_{e} / c\right)$. Expanding $\Omega(s)$ near the cyclotron resonance,

$\Omega(\varepsilon) \simeq \omega\left(1+\frac{s}{L_{s}}\right)$

where $L_{s}$ is the scale length for magnctic field gradients along the ray, the integral over $\operatorname{Im}\left\{z[\xi(s)]^{-1}\right\}$ can be expressed 10

$\int_{-\infty}^{\infty} \mathrm{ds} \operatorname{Im}\left\{\frac{1}{\mathrm{Z}[\xi(s)]}\right\}=\frac{\pi}{2}, \quad \mathrm{n}=\cos \theta \mathrm{L}_{\mathrm{s}} \mathrm{v}_{\mathrm{e}} / \mathrm{c}$

The total damping coefficient can be expressed

$\alpha_{T}=\frac{4 \pi^{2} \frac{\omega v_{e}^{2}}{c \frac{e}{c} L_{s} n_{ \pm}^{2} \Lambda_{I}\left(n_{ \pm}^{2}\right)}}{P \frac{\partial \Lambda_{0}}{\partial n_{ \pm}} \sin ^{2} \theta}=2 \pi s / 2-\frac{\omega v_{c}}{c} n_{ \pm} \operatorname{rns} \theta T_{s} n_{i}(s=0, \theta)$,

where $\mathrm{n}_{i}(s=0, \theta)$ is the local damping rate evaluated exactly at the cyclotron layer. Eldridge, Namkung, and England 10 derived a similar equation and applied it to perpendicularly stratified plasmas of tokamak. interest.

Considering plane stratified plasmas, it can be seen from Fig. 4 that the total power deposited depends strongly upon the direction of stratification. For perpendicular stratification with magnetic field scale length 
$\mathrm{L}_{\mathrm{x}}$ the segment of the ray inside the resonant layer decreases as $\theta$ increases and the magnetic scale length along the ray is given by $L_{s}=$ $L_{x} / \sin \theta$. For parallel stratification with magnetic field scale length $\mathrm{L}_{z}$ the segment of the ray inside the resonant layer increases as $\theta$ increases, so that the magnetic scale length along the ray is given by $\mathrm{L}_{\mathrm{s}}=\mathrm{L}_{\mathrm{z}} / \cos \theta$.

Figure 7 shows the fraction of power absorbed from a ray, $f[E q$. (33)], as a function of propagation angle $\theta$ for typical EBT-I plasma parameters; $\omega / 2 \pi=18 \mathrm{GHz}, \mathrm{T}_{\mathbf{e}}=300 \mathrm{eV}$, and $\omega_{\mathrm{pe}}^{2} / \omega^{2}=0.3-0.7$. The magnetic field scale length was taken to be $L_{x}=L_{z}=10 \mathrm{~cm}$ for both directions of stratification. This is characteristic of the gradients near the fundamental resonant zone at the minor axis. Figures $7(a)$ and $7(b)$ are for the ordinary mode with perpendicular and parallel stratification, respectively. It can be seen that the fractional power absorbed increases with increasing density and with propagation angle. It is much greater for the parallel stratified plasma. The important point is that ordinary mode absorption is very weak at the temperatures and densities characteristic of EBT-I. Even for the case of parallel stratification at the highest density, the fractional absorption exceeds a $f$ ew percent only for $\theta$ very near $90^{\circ}$. Figure $7(\mathrm{c})$ shows extraordinary mode absorption for a perpendicularly stratified plasma. The fractional power absorption decreases with increasing density and propagation angle. As previously discussed, the extraordinary mode is totally absorbed in a parallel stratified plasma when propagating from the accessible side. However, even for perpendicular stratification, the extraordinary mode is very heavily damped at EBT-I densities and temperatures.

Figure 8 shows similar plots of fractional power absorption for projected EBT-II parameters: $\omega / 2 \pi=120 \mathrm{GHz}, \mathrm{T}_{\mathrm{e}}=2 \mathrm{keV}$, and $\omega_{\mathrm{pe}}^{2} / \omega^{2}=$ 0.3-0.7. The magnetic field scale length was again taken as $\mathrm{L}_{\mathrm{x}}=\mathrm{L}_{z}=$ $10 \mathrm{~cm}$. For EBT-II parameters the ordinary mode absorption is quite significant for both directions of stratification. The extraordinary mode is almost completely absorbed except for $\theta$ near $90^{\circ}$.

Although the basic physics of the cyclotron heating processes is comparatively well understood, a number of important effects have been 

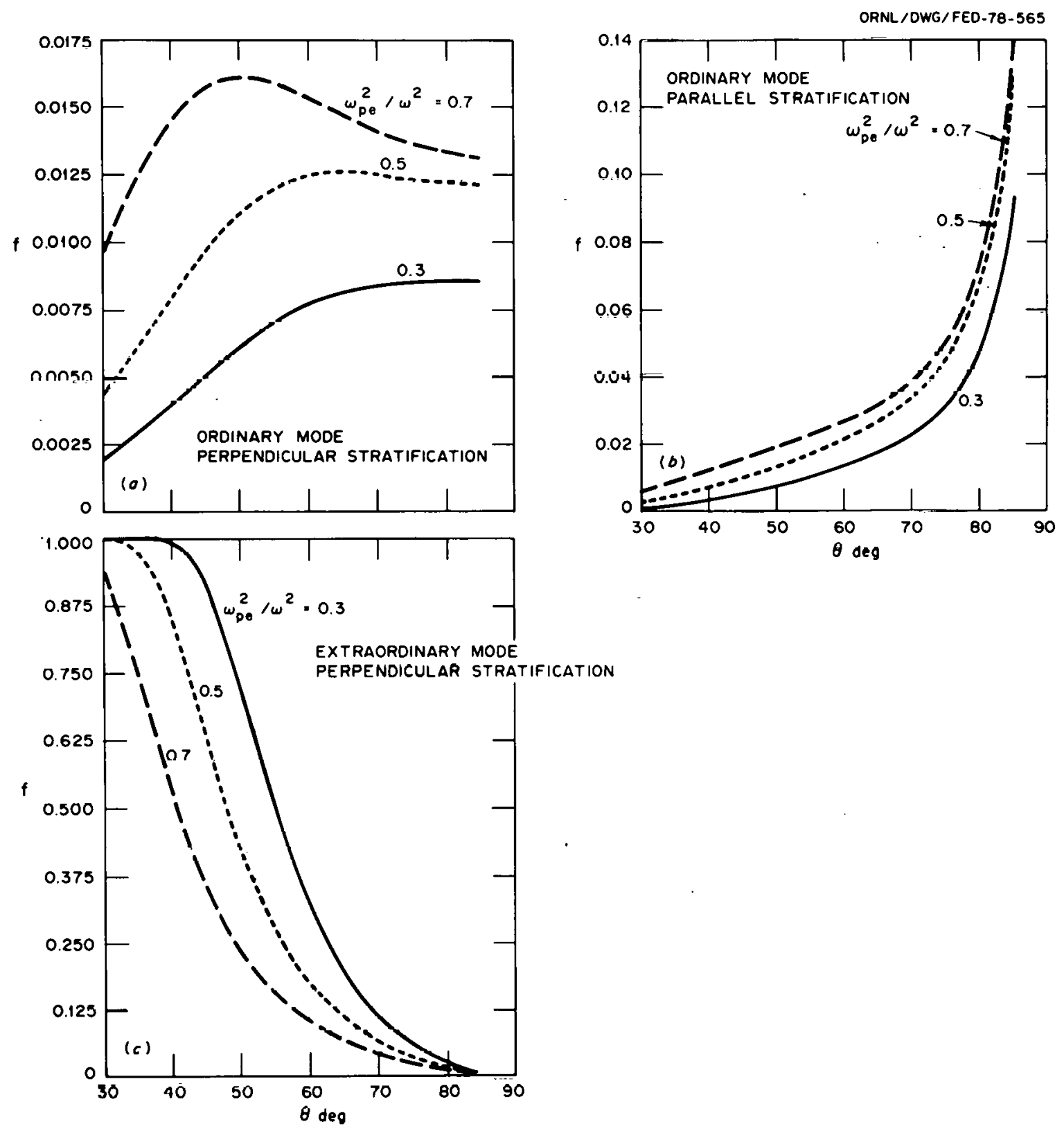

F1g: 7. liraction ol puwer absorbed from a ray in passing through cyclotron resonance at angle $\theta$ for various values of $\omega_{\text {pe }}^{2} / \omega^{2}$. Plasma
parameters are characteristic of EBT-I. 

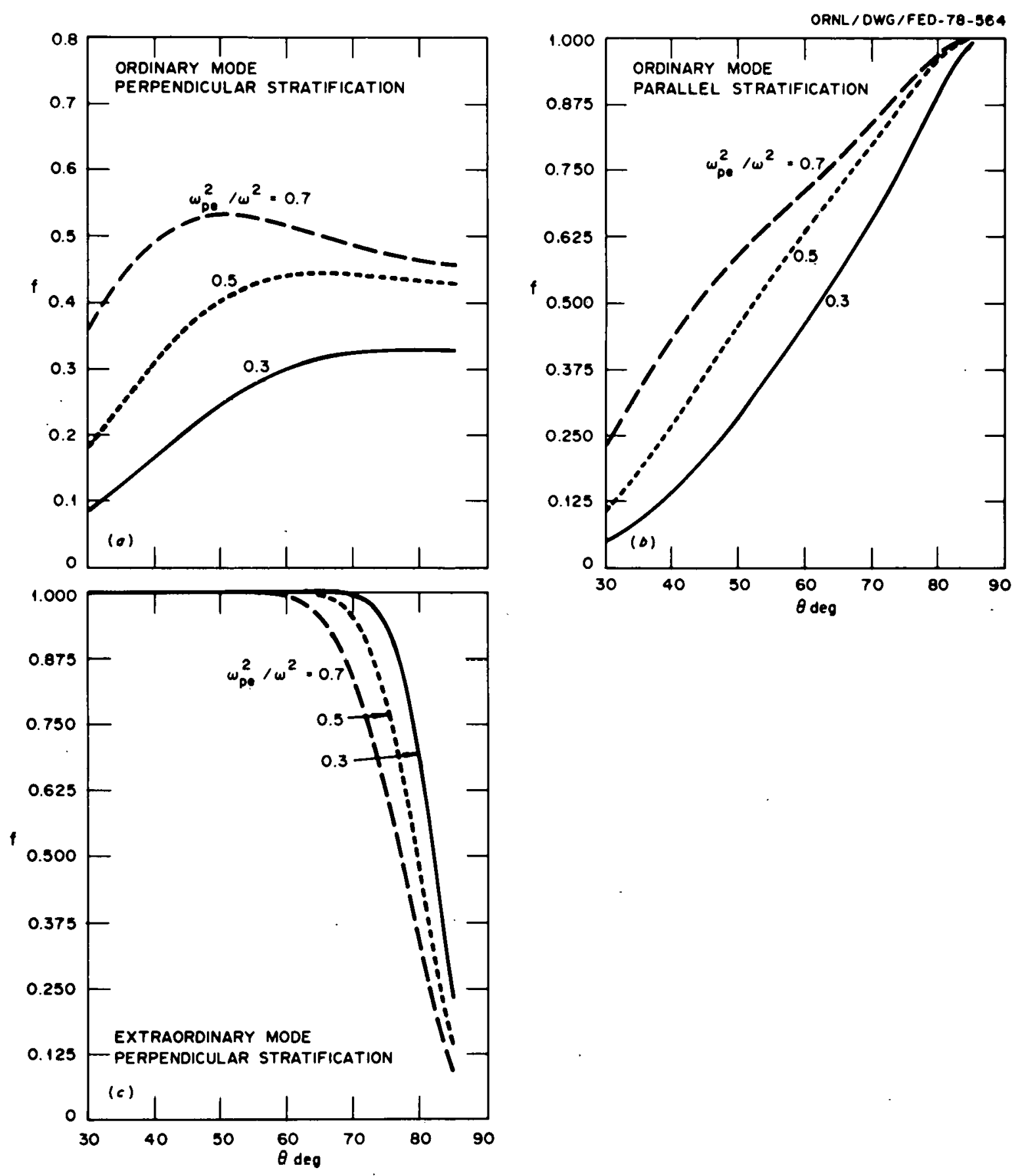

Fig. 8. Fraction of power absorbed from a ray in passing through cyclotron resonance at angle $\theta$ for various values of $\omega_{\mathrm{pe}}^{2} / \omega^{2}$. Plasma parameters are characteristic of projections for EBT-II. 
omitted from the absorption calculations up to now. In particular, the inclusion of damping at second and higher cyclotron harmonics and absorption by the hot electron annulus are matters of top priority. An alternative method of computing energy deposition is to make use of the Hermitian part of the plasma conductivity $\underset{\approx}{\stackrel{\mathrm{H}}{\sigma}}$ in the energy transport equation 15

$\frac{\mathrm{du}}{\mathrm{dt}}=-\mathrm{u} \underset{\sim}{\nabla} \cdot \frac{\partial \Omega}{\partial \mathrm{k}}-{\underset{\sim}{\mathrm{E}}}^{*} \cdot \underset{\approx}{\sigma} \cdot \underset{\sim}{\mathrm{H}}$,

where

$$
\begin{aligned}
& u=\frac{1}{8 \pi} \underset{\sim}{E^{*}} \cdot \frac{\lambda(\underset{\sim}{(\omega)})}{\partial \omega} \cdot \underset{\sim}{E}=\text { total wave energy, } \\
& \frac{\partial \Omega}{\partial \underset{\sim}{k}}=\underset{\sim g}{v}=\text { group velocity, and } \\
& \underset{\sim}{E}=\text { plasma dielectric tensor. }
\end{aligned}
$$

The first term on the right of Eq. (37) represents geometric focusing and defocusing as well as speeding up and slowing down of the group velocity. The second term describes energy dissipation. The RAYS code is being adapted to calculate the electric field strength by this method. Another area which has not yet been considered is the effect on energy absorption of quasi-linear distortion of the distribution function. The toroidal core plasma is expected to remain nearly Maxwellian because of collisions but quasi-linear effects may be very important for the hot electron component. 
3. RAY TRACING IN THREE DIMENSIONS: THE RAYS CODE

If the position $\underset{\sim}{r}$ and propagation vector $\underset{\sim}{k}$ of the microwave field are specified, the results of Sect. 2 can be used to calculate the local rate of energy deposition. However, for a general non-plane stratified medium the propagation regions and values of $\mathrm{k}$ as a function of space cannot be determined in a straightforward way. This information can be obtained by the method of geometrical optics. Much of the effort in the microwave heating study has gone into the development of a ray tracing computer code, RAYS, capable of operating with a wide variety of plasma equilibrium configurations. It has been written in a modular form so that equilibrium geometries and wave dispersion relations are readily interchangeable. As of this writing the RAYS code calculated ray paths, local wave number $\underset{\sim}{\mathrm{k}}$, and local electric field polarization using the Appleton-Hartree dispersion relation, as well as fractional power deposition using the finite temperature dispersion relation (Sect. 2).

The equations solved to obtain the ray trajectories are the Hamiltonian form of the geometrical optics equations (see, for example, Refs. 13 and 15). For general space- and time-varying media, the waves satisfy a dispersion relation of the form

$D(\underset{\sim}{k}, \omega, \underset{\sim}{r}, t)=\operatorname{det} \underset{\sim}{(\varepsilon)}=0$,

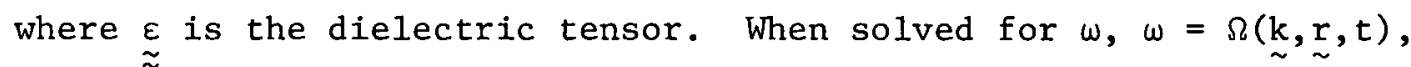
the dispersion relation plays the role of the Hamiltonian defining the trajectories $\underset{\sim}{r}(t)$ and $\underset{\sim}{k}(t)$

$\frac{d \underset{\sim}{\mathrm{r}}}{\mathrm{dt}} \equiv \underset{\sim}{\mathrm{v}}=\frac{\partial \Omega}{\underset{\sim}{\partial \mathrm{k}}} \quad$ or $\quad \underset{\sim g}{\underset{\sim}{v}}=-\frac{\partial \mathrm{D} / \partial \mathrm{z}}{\partial \mathrm{D} / \partial \omega}$,

$\frac{d \underset{\sim}{d t}}{d t}=-\frac{\partial \Omega}{\partial \underset{\sim}{r}}$ or $\quad \frac{d \underset{\sim}{d t}}{d t}=\frac{\partial \mathrm{D} / \partial r}{\partial \mathrm{D} / \partial \omega}$.

For time-invariant media $(\partial / \partial t=0)$, the arc length along the ray, $s$, is a convenient parameter and the above equations can be reduced to 


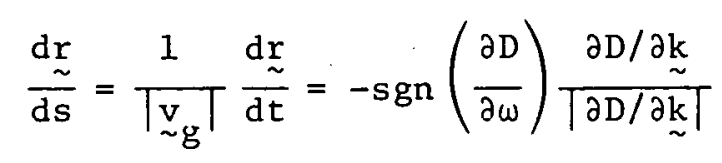

$\frac{\mathrm{dk}}{\mathrm{ds}}=\frac{1}{\underset{\sim}{\mathrm{v}} \mid} \frac{\mathrm{dk}}{\mathrm{dt}}=\operatorname{sgn}\left(\frac{\partial \mathrm{D}}{\partial \omega}\right) \frac{\partial \mathrm{D} / \partial \underset{\sim}{\mathrm{r}}}{|\partial \mathrm{D} / \underset{\sim}{\mathrm{k}}|}$

Equations (41) and (42) are the ones integrated in the tracing code. The Appleton-Hartree dispersion relation, 13,18 a high frequency limit $\left(\omega \gg \Omega_{i}\right)$ of the cold plasma dispersinn relation, takes the form

$\mathrm{D}(\underset{\sim}{\mathrm{k}}, \omega, \underset{\sim}{\mathrm{r}})=1-\frac{c^{2} \mathrm{k}^{2}}{\omega^{2}}-\frac{2 \alpha(1-\alpha)}{?(1-\alpha)-\beta \operatorname{oin}^{2} \theta \pm \Gamma\left(0, \frac{1}{\sim}\right)}$

where

$$
\begin{aligned}
\Gamma(\omega, \theta, \underset{\sim}{r}) & =\left[\beta^{2} \sin ^{4} \theta+4 \beta(1-\alpha)^{2} \cos ^{2} \theta\right]^{1 / 2}, \\
\alpha(\omega, \underset{\sim}{r}) & =\omega_{p e}^{2}(\underset{\sim}{r}) / \omega^{2}, \text { and } \\
\beta(\omega, \underset{\sim}{r}) & =\Omega_{e}^{2}(\underset{\sim}{r}) / \omega^{2} .
\end{aligned}
$$

The $+(-)$ sign in Eq. (43) corresponds to the ordinary mode (extraordinary mule) of propagation.

The group velocity is most conventently calculated in a local. coordinate system with $\hat{z}$ taken along the local magnetic field and $\hat{x}$ taken in the $\underset{\sim}{B}, \underset{\sim}{k}$ plane (Fig. 9). The group velocity is resolved into components along $\hat{k}=k /|k|$ and $\hat{\theta}$,

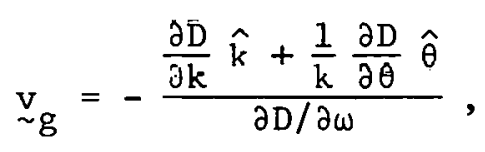




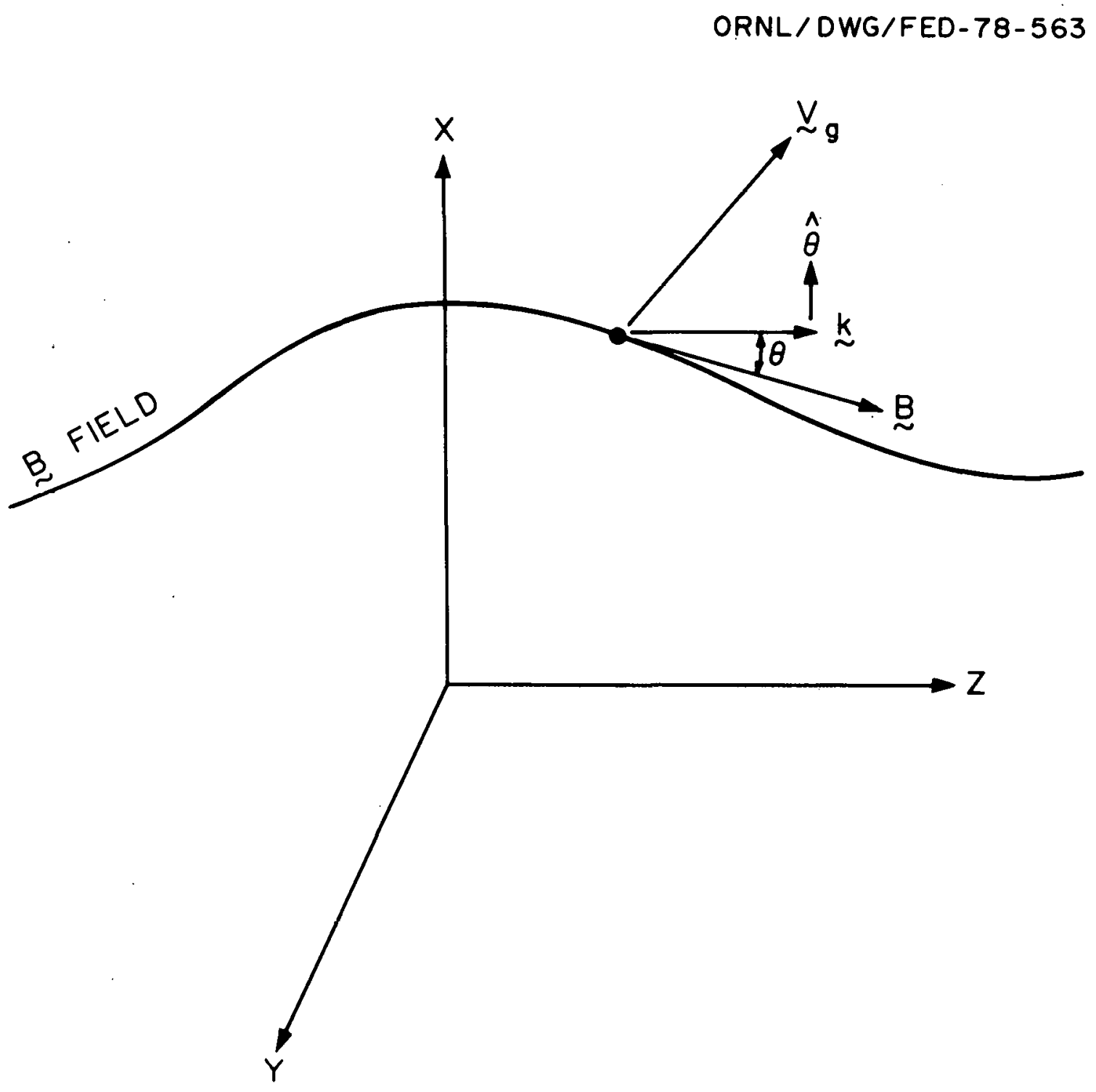

Fig. 9. Local magnetic field coordinate system used in ray tracing. 
where

$$
\hat{\theta}=\frac{\underset{\sim}{k} \times(\underset{\sim}{k} \times \underset{\sim}{\mathrm{B}})}{\mid \underset{\sim}{\mathrm{k}} \times \underset{\sim}{\mathrm{k}} \times \underset{\sim}{\mathrm{B}}) \mid}
$$

Using these expressions in Eq. (39), we obtain

$$
\frac{\mathrm{dr}}{\mathrm{ds}}=-\operatorname{sgn}\left(\frac{\partial D}{\partial \omega}\right) \frac{\frac{\partial D}{\partial k} \hat{k}+\frac{1}{k} \frac{\partial D}{\partial \theta} \hat{\theta}}{\left(\frac{\partial D}{\partial k}\right)^{2}+\frac{1}{k^{2}}\left(\frac{\partial D}{\partial \theta}\right)^{2}} .
$$

The right-hand sides of Eqs. (42) and (45) can be obtained analytically by a straightforward but tedious differentiation of Eq. (43). Expressing the dispersion relation $D(\omega, \underset{\sim}{\mathrm{k}}, \underset{\sim}{\mathrm{r}})$ as

$\left.D=1-\frac{k^{2} c^{2}}{\omega^{2}}-G[\alpha \underset{\sim}{r}, \omega), \beta(\underset{\sim}{r}, \omega), \Gamma(\underset{\sim}{r}, \omega, \theta), \theta\right]$,

where

$$
G(\alpha, \beta, \Gamma, \theta) \equiv \frac{N(\alpha)}{D_{ \pm}(\alpha, \beta, \Gamma, \theta)} \equiv \frac{2 \alpha(1-\alpha)}{2(1-\alpha)-\beta \sin ^{2} \theta \pm \Gamma(\alpha, \beta, \theta)} .
$$

we have

$\frac{\partial \mathrm{D}}{\partial \mathrm{k}}=-\frac{2 \mathrm{~kg} \mathrm{c}^{2}}{\omega^{2}}$

$\frac{\partial D}{\partial \theta}=\frac{N}{D_{ \pm}^{2}} \frac{\partial D_{ \pm}}{\partial \theta}=-\frac{2 N \sin \theta \cos \theta}{D_{ \pm}^{2}}\left[\beta \pm \frac{\beta^{2} \sin ^{2} \theta-2 \beta(1-\alpha)^{2}}{\Gamma}\right]$,

$\frac{\partial D}{\partial \omega}=\frac{2 k^{2} c^{2}}{\omega^{3}}+\frac{2 \alpha}{\omega}\left(\frac{\partial G}{\partial \alpha}+\frac{\partial G}{\partial \Gamma} \frac{\partial \Gamma}{\partial \alpha}\right)+\frac{2 \beta}{\omega}\left(\frac{\partial G}{\partial \beta}+\frac{\partial G}{\partial \Gamma} \frac{\partial \Gamma}{\partial \theta}\right)$,

$\underset{\sim}{\frac{\partial D}{\partial r}}=-\left(\frac{\partial G}{\partial \alpha}+\frac{\partial G}{\partial \Gamma} \frac{\partial \Gamma}{\partial \alpha}\right) \underset{\sim}{\nabla \alpha}-\left(\frac{\partial G}{\partial \beta}+\frac{\partial G}{\partial \Gamma} \frac{\partial \Gamma}{\partial \beta}\right) \nabla \beta$ 
$\frac{\partial G}{\partial \alpha}+\frac{\partial G}{\partial \Gamma} \frac{\partial \Gamma}{\partial \alpha}=\frac{1}{D_{ \pm}^{2}}\left[2(1-2 \alpha) D_{ \pm}+2 N \pm N \frac{4 \beta(1-\alpha)}{\Gamma} \cos ^{2} \theta\right]$

$\frac{\partial G}{\partial \beta}+\frac{\partial G}{\partial \Gamma} \frac{\partial \Gamma}{\partial \beta}=\frac{N}{D_{ \pm}^{2}}\left[\sin ^{2} \theta \pm \frac{\beta \sin ^{4} \theta+2(1-\alpha)^{2} \cos ^{2} \theta}{\Gamma}\right]$

$\underset{\sim}{\nabla \alpha}=\alpha \frac{\nabla \mathrm{n}_{0}}{\mathrm{n}_{0}}$

$\underset{\sim}{\nabla \beta}=2 \beta \underset{\sim}{\stackrel{\nabla}{|\overrightarrow{\mid}| B \mid} .}$

The unit vectors in Eqs. (42) and (45) are expressed in terms of a Cartesian coordinate system with origin located at the origin of the model equilibrium. These equations are then numerically integrated to obtain the Cartesian components of $\underset{\sim}{r}$ and $\underset{\sim}{k}$ as functions of arc length $s$ and the initial conditions of the ray $\underset{\sim}{r}{ }^{0}$ and $\underset{\sim}{\mathrm{k}}$.

The RAYS code has been run and tested extensively in (1-D) slab geometry and (2-D) cylindrical or quasi-toroidal geometry. For example, the ray paths can be calculated analytically for the perpendicular incidence of the ordinary mode on a plasma cylinder with a parabolic density profile.19 Rays calculated using the code agree exactly with the analytic results for this case.

Figures 10 and 11 show extraordinary mode rays in a quasi-toroida1 geometry. The equilibrium is a straight cylinder having a magnetic field in the $z$ direction which varies such that $\Omega_{c}(x)=\omega(1+x / L)^{-1}$. The density profile was taken to be a parabolic function of radius, $\omega_{p e}^{2}(x)=\left(\omega_{j e}^{0}\right)^{2}\left(1-r^{2} / a^{2}\right)$. Figure $10(a)$ shows a projection of the rays onto a cross section $(x-y)$ of the plasma column, while Fig. 10(b) shows a projection onto the equatorlal $(x-z)$ plane. The propagation is from the inner or high magnetic field side of the equilibrium at various angles with respect to the field direction. Waves injected from the outer or low field side of the equilibrium are reflected at 

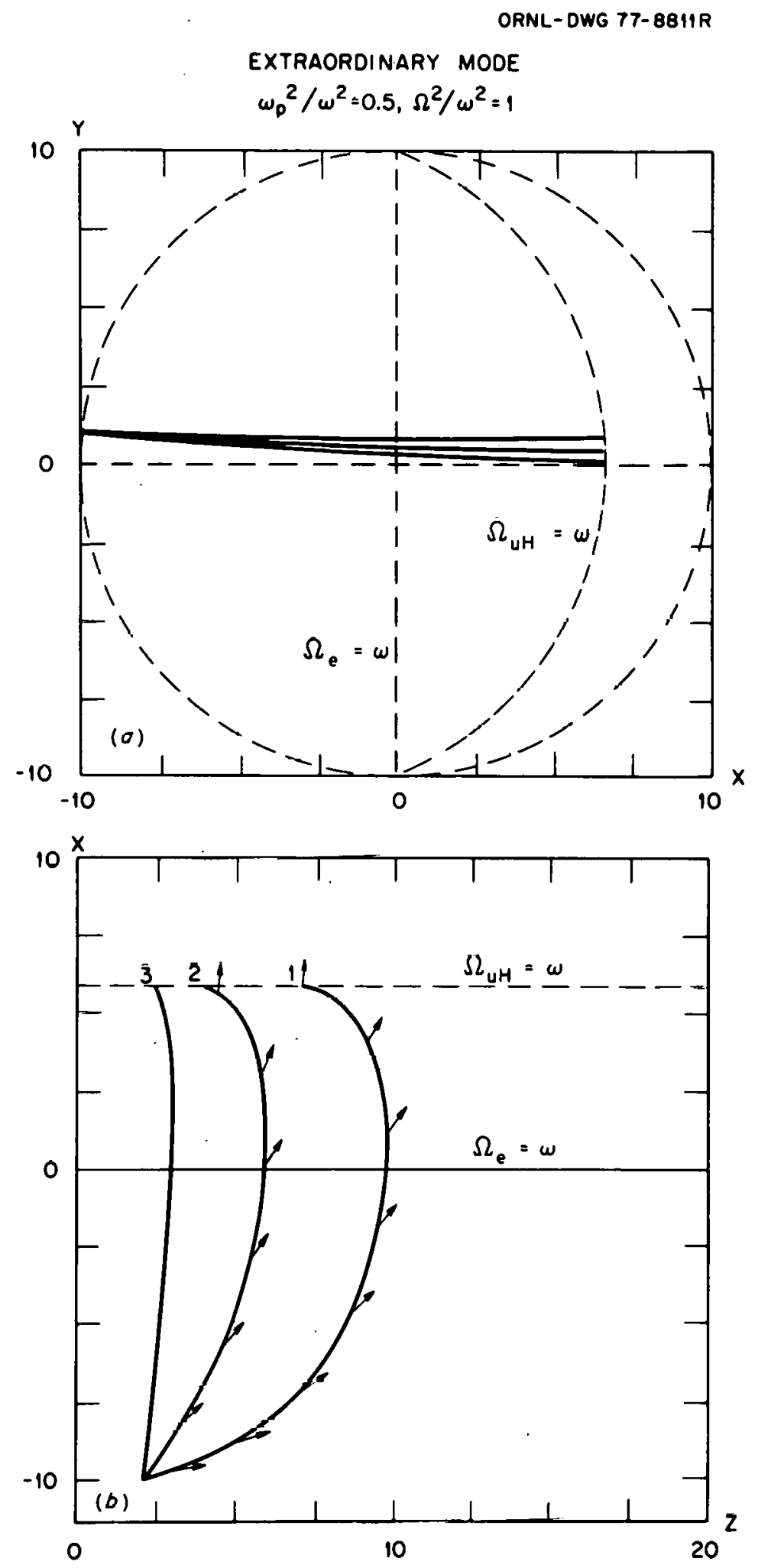

Fig. 10. Extraordinary mode rays injected from the high magnetic field side in a quasitoroidal geometry, low density case $\omega_{\text {pe }}^{0} / \omega^{2}<1$. Vectors (b) indicate the direction of $\underset{\sim}{k}$ at various points along the rays. 

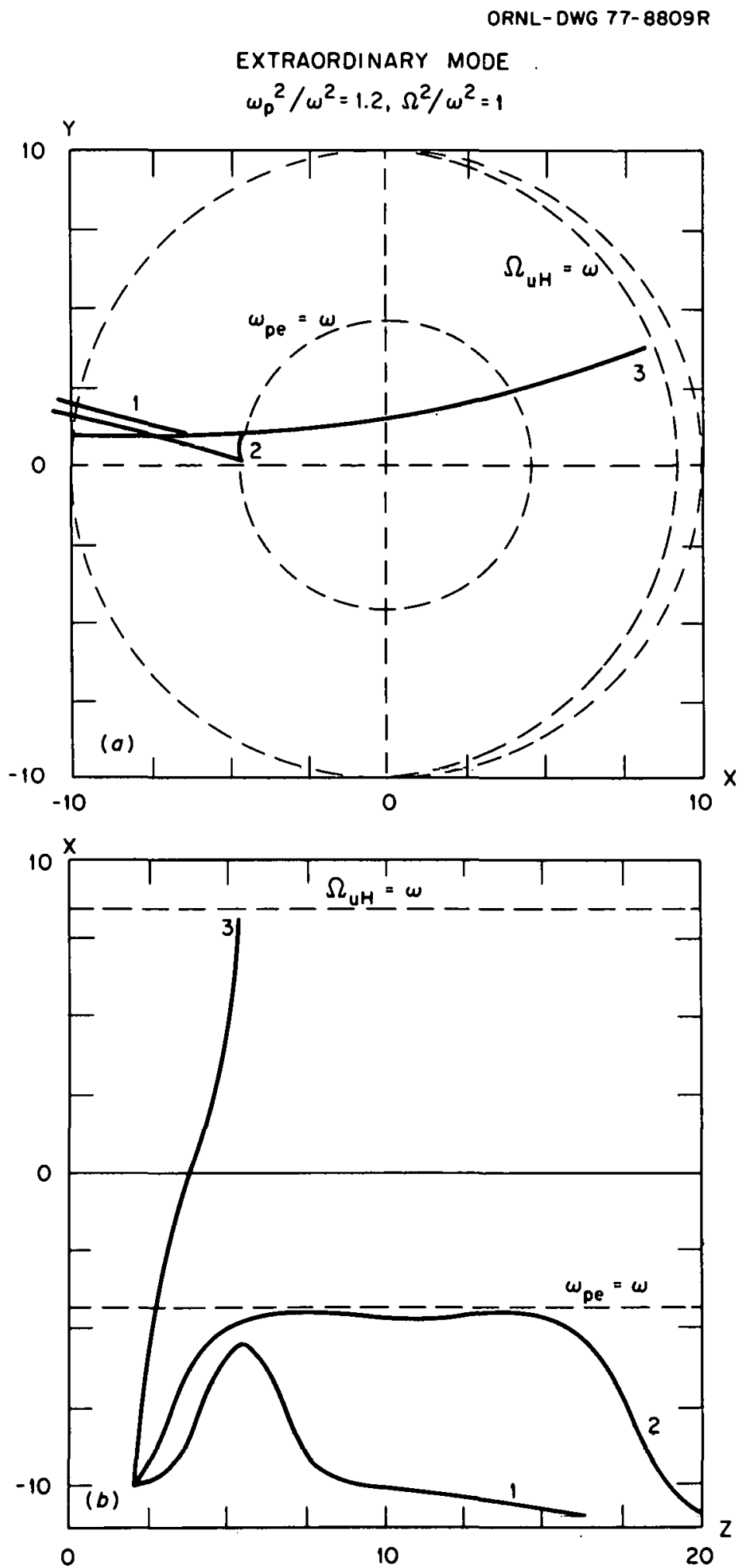

Fig. 11. Extraordinary mode rays injected from the high magnetic field side in a quasitoroidal geometry, high density case $\omega_{\mathrm{pe}}^{0} / \omega^{2}>1$. 
the right-hand cutoff. In Fig. 10 the peak density was chosen such that $\left(\omega_{\mathrm{pe}}^{0}\right)^{2} / \omega^{2}=0.5$. The rays propagate past the cyclotron resonance layer $(x=0)$ to the upper hybrid resonance. As. the rays approach the upper hybrid resonance, the group velocity becomes parallel to the magnetic field while $\underset{\sim}{\mathrm{k}}$ [the vectors shown in Fig. $10(\mathrm{~b})$ ] becomes perpendicular to B. This behavior is characteristic of perpendicularly stratified media. Figure 11 is similar to Fig. 10 except that the peak density was chosen such that $\left(\omega_{\mathrm{pe}}^{0}\right)^{2} / \omega^{2}=1.2$. In this case only rays having small initial values of $k_{z}$ can penetrate to the upper hybrid resonance layer. Notice the double cusp nature of the $x-y$ projection of ray 2 .

Recently the 3-D RAYS code has been run using a model axisymmetric mirror equilibrium (described in Sect. 2.1). Figure 12 shows the results of Injecting extraordinary mode rays at varying angles into a mirror equilibrium from a point located at the mirror midplane $(z=0)$ and just above the equatorial plane $(y=0)$. The plasma parameters were chosen to be representative of EBT-I: $\omega / 2 \pi=18 \mathrm{GHz},\left(\omega_{\mathrm{pe}} / \omega\right)_{\max }=0.5$, and $v_{e} / c=0.034$. The density profile was modeled to be constant along field lines and to decrease in moving radially away from the z-axis. Figure 12 shows the projection of the ray path onto the equatorial plane and the wave vectors $\underset{\sim}{k}$ at various points along the ray. Also shown in Fig. 12 are the magnetic field lines and equatorial cross sections of the cyclotron resonant surface and right-hand cutoff surface. It can be seen that the rays propagating from the low magnetic field region near the mirror midplane do not penetrate to the cyclotron resonance surface but are reflected at the right-hand cutoff.

There are a number of mechanisms by which the extraordinary mode could eventually be absorbed by the plasma: (1) by Budden tunneling through the right-hand cutoff to the resonance layer, (2) by linear mode conversion to a warm plasma mode and subsequent absorption by Landau damping, and (3) by a complicated process involving multiple reflections, depolarization into the ordinary mode, reconversion to extraordinary mode on the high field side of the cyclotron resonant zone, and absorption at the resonant layer. The theory of these three processes is in too primitive a state to consider including them in the RAYS code at 


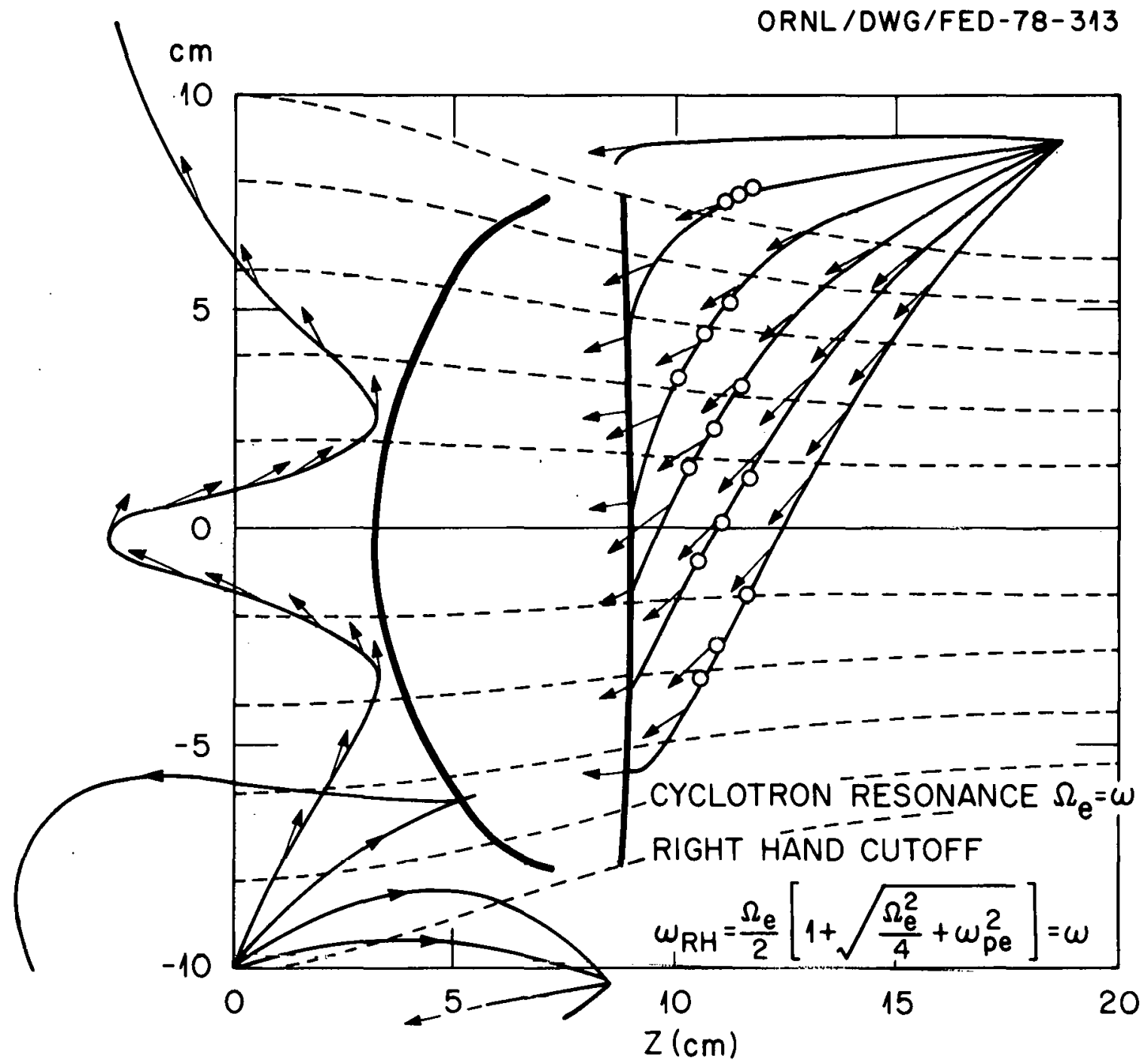

Fig. 12. Extraordinary mode rays propagating in a mirror plasma characteristic of EBT-I. Circles mark 1\%, 50\%, and $99 \%$ absorption. Vectors indicate the direction of $\mathrm{k}_{\sim}$. 
this time. However, we can examine the fate of an extraordinary mode ray originating near the mirror throat which, for example, might arise from depolarization of an ordinary mode ray on reflection. Figure 12 also shows ray paths and wave vectors for extraordinary mode rays injected at varying angles in the equatorial plane from a point near the mirror coil. On this side, the cyclotron resonance zone is accessible. It can be seen that the rays approach the cyclotron resonance layer with $v_{g}$ nearly perpendicular to $\underset{\sim}{\mathrm{B}}$ and $\underset{\sim}{\mathrm{k}}$ nearly parallel to $\underset{\sim}{\mathrm{B}}$. This is characteristic of parallel stratified media in the cold plasma model. The asterisks on the rays mark the points at whirh $1 \%, 50 \%$, and $99 \%$ of the wave power lids been absorbed. For the F.RT-T narameters unce in the calculation, the waves are completely damped before reaching the resonant layer. The ray paths show a tendency to focus the energy toward the center of the plasma. However, this can hardly be considered significant due to the early damping of the wave energy.

The situation is much less complicated for the ordinary mode. As long as the microwave frequency is higher than the plasma frequency, the entire plasma is accessible to the ordinary mode. Figure 13 shows ordinary mode rays for the same equilibrium and injection geometry as in Fig. 12. The rays are virtually straight with olight curvature due to density gradients.

For the EBT-I parameters 11sed; nnly raye within a nariuw cone liave mure than $1 \%$ of the wave energy ahsnrbed in paoding through the cyclutron resonance (the circles on the rays mark the location at which $1 \%$ of the wave energy has been absorbed). Despite its small damping rate, the ordinary mode may play an important role in the heating of EBT-I since conversion of ordinary mode energy in the high field region can be an 1mportant source of extraordinary mode which subsequently propagates to the cyclotron resonance.

Fui d plasma having parameters characteristic of the projected values for EBT-II, the ordinary mode is heavily damped. Figure 14 shows the result of injecting ordinary mode rays at varying angles into a plasma with $\omega / 2 \pi=120 \mathrm{GHz},\left(\omega_{\mathrm{pe}} / \omega\right)_{\max }=0.5$, and $v_{\mathrm{e}} / \mathrm{c}=0.09$. The rays are again virtually straight with slight curvature due to density 


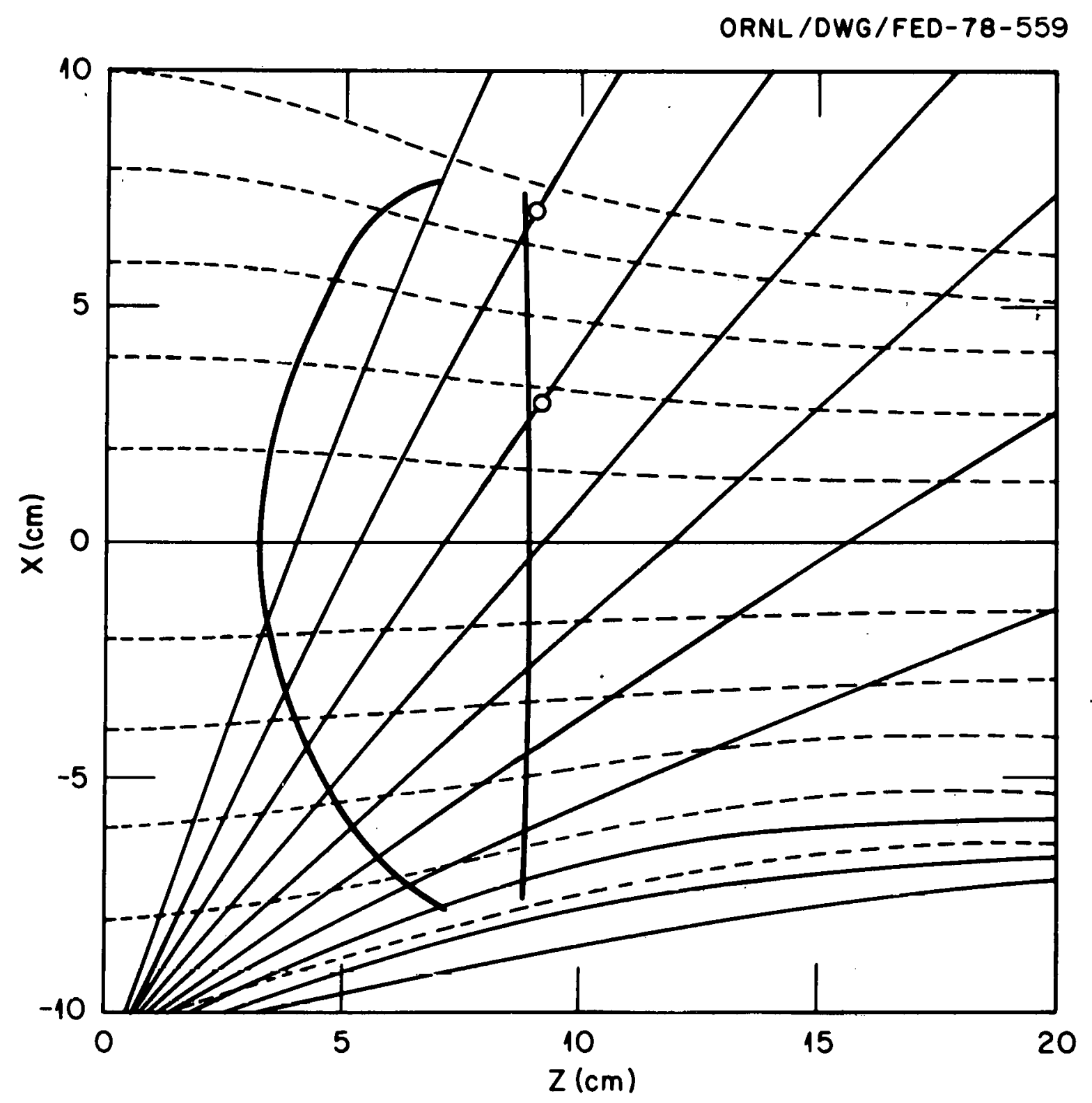

Fig. 13. Ordinary mode propagation in a mirror plasma characteristic of EBT-I. Circles mark $1 \%$ absorption. No rays had more than $2 \%$ power absorbed. 


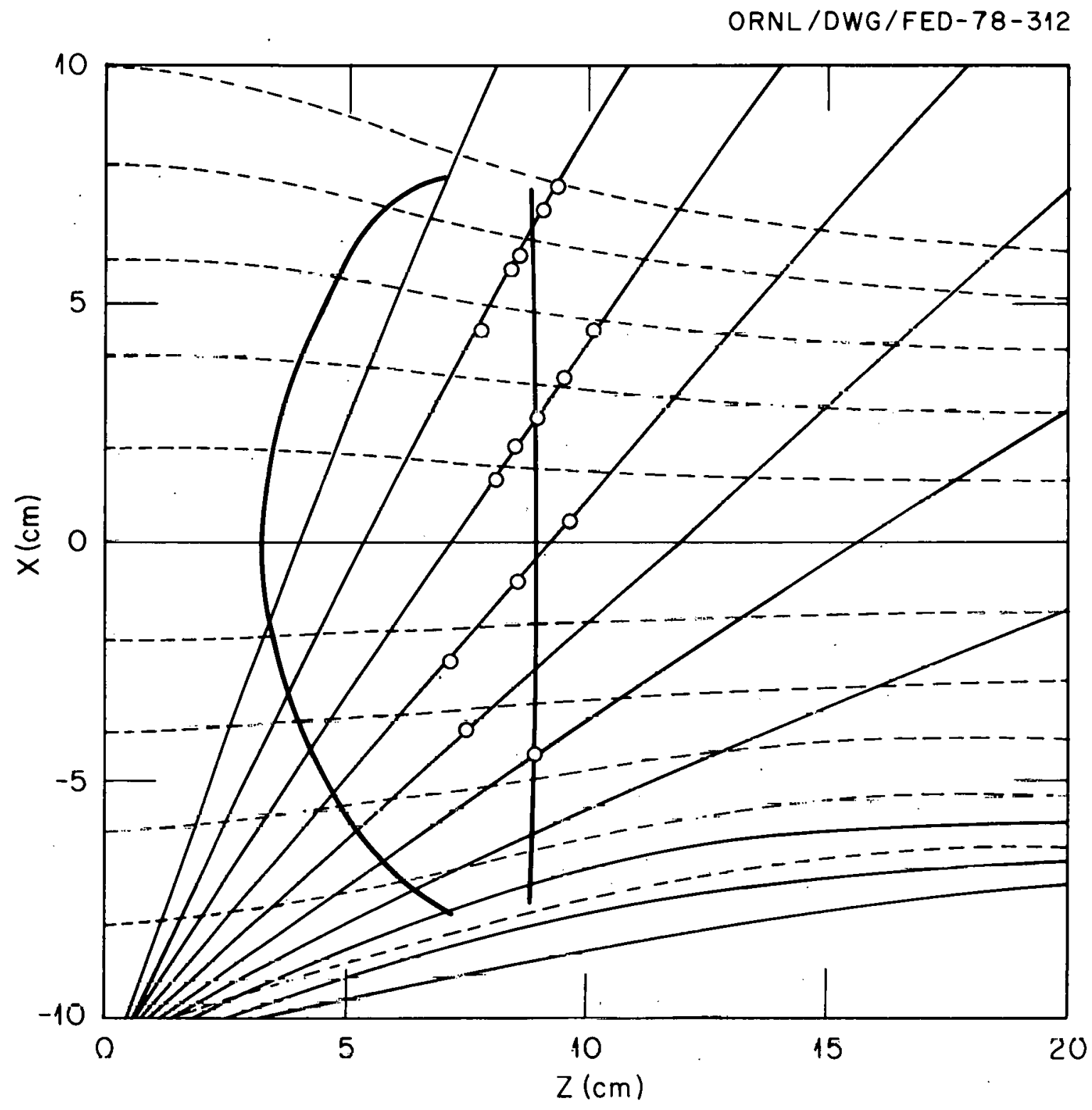

Fig. 14. Ordinary mude propagation in a plasma characteristic of EBT-II. Circles mark $1 \%, 10 \%, 20 \%, \ldots$ power absorption. 
gradients. The cross marks indicate the point at which $1 \%, 10 \%, \ldots$ of the wave energy has been absorbed. In this case, up to $60 \%$ of the energy is absorbed in a single pass through the resonant zone.

A number of tasks remain to be done in this area of ray tracing theory, and a great deal of additional coding will be required before detailed calculation of heating profiles can be carried out with the RAYS code. For example, work is currently under way to include the effects of finite temperature on the ray paths. Provision for reflection, partial absorption, and partial depolarization of rays at conducting walls needs to be included in the code. This may be particularly important in understanding complicated absorption processes of the extraordinary mode in EBT-I. If a ray approaches a cutoff layer at an angle near perpendicular, the ray may not have a continously turning tangent but may have a cusp or "spitze." The Hamiltonian form of the equations cannot track such a nondifferentiable path. An alternative set of equations which can follow a cusp needs to be provided; for example, the Booker quartic formulation. Realistic, finite beta, toroidal equilibrium field and density models need to be adapted for use in the RAYS code. 


\section{JOINING RELATION AT REGIONS OF GEOMETRICAL OPTICS BREAKDOWN}

The geometrical optics approximation becomes invalid in any region for which the wave refractive index vanishes, becomes infinite, or changes on a spatial scale length smaller than the wavelength. In such regions a full wave treatment must be employed in order to determine the fate of incoming microwave energy. There are several processes which can occur: (1) the wave can be reflected; (2) if the evanescent region is thin, the energy can penetrate by Budden tunneling; ( 3 ) the wave can be absorbed directly by coupling to the plasma particles; and (4) the wave can be converted to other plasma modes.

A large body of literature exists in which special cases of each of the above processes have been studied. However, the analysis is so - involved and so specialized that the results cannot be readily adapted for use in the computer code under development. As an example, the propagation of a plane wave vertically incident on a horizontally stratified plasma with constant magnetic field and linear density gradient is a classic problem or ionospheric physics. However, even this simplified problem has not been solved for arbitrary angle of incidence. For a plane stratified, cold plasma model, one must, in general, solve a fourth order differential equation for the fields in regions where geometrical optics breaks down. In a finite temperature plasma which allows coupling to warm plasma modes, the system is at least sixth order. It is clear that a great deal of analysis must yet be done in order to develop connection formulas which are sufficiently flexible to be used in the computer code.

The processes which seem most likely to play an important role in EBT are: (1) reflection and Budden tunneling at the right-hand cutoff, (2) conversion between ordinary and extraordinary modes of propagation, and possibly (3) conversion of the extraordinary mode waves to warm plasma electrostatic waves at the second harmonic cyclotron resonance.

For illustration, Fig. 15 shows the location of the first and second harmonic cyclotron resonance zones and the right-hand cutoff zones for $18-\mathrm{GHz}$ extraordinary mode radiation in a model EBT-I equilibrium. The magnetic equilibrium has an annular beta of $40 \%$ and a density 
ORNL/DWG/FED-78-311

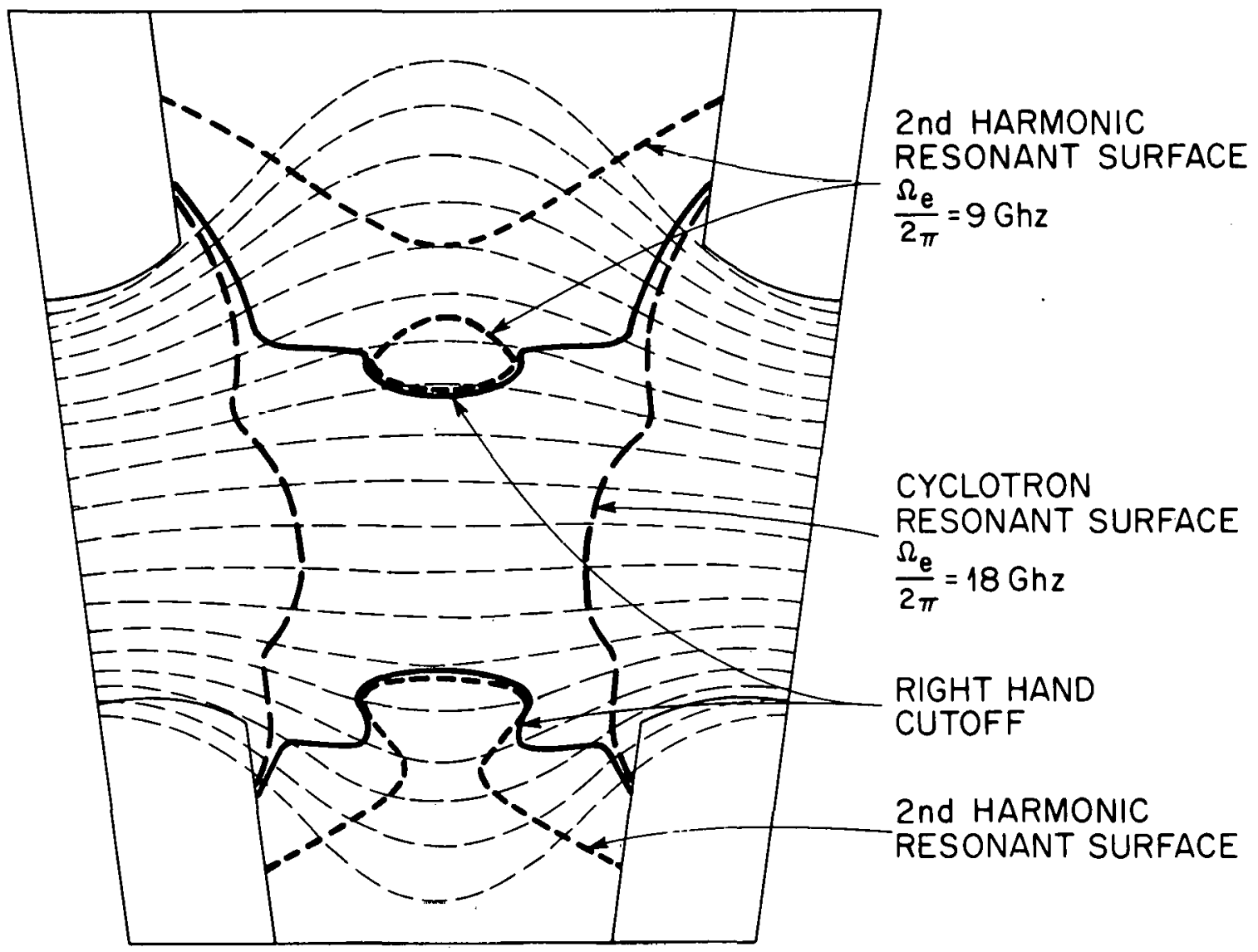

Fig. 15. Location of first and second harmonic cyclotron resonance zones and the right-hand cutoff zones for $18-\mathrm{GHz}$ extraordinary mode radiation in a model EBT-I equilibrium. 
profile which is nearly flat inside the annulus and drops rapidly in the outer region at the annulus $(\nabla B>0)$ to 0.1 of its peak value. The peak density was chosen to be $\mathrm{n}_{0}=2 \times 10^{12} / \mathrm{cm}^{3}\left(\omega_{\mathrm{pe}}^{0} / \omega=0.707\right)$. With these parameters the fundamental cyclotron resonance zone is completely cut off from waves propagating from near the mirror midplane. The second harmonic resonance is accessible, but for higher density the right-hand cutoff moves radially outward to remove a portion of the second harmonic resonance from the accessible region. At this density the entire plasma is accessible to the ordinary mode.

Within the geometrical optics approximation an extraordinary mode ray is reflected near the right-hand cutoff when propagating from the. low magnetic field or low density side of the cutoff layer. The actual point of reflection depends on the plasma geometry (stratification) and the angle of wave propagation [see, for example, Eqs. (13) and (17)]. Exactly at the right-hand cutoff $\omega_{\mathrm{RH}}=\omega$, where

$\omega_{\mathrm{RH}}=\frac{\Omega_{\mathrm{e}}}{2}+\left(\frac{\Omega_{\mathrm{e}}^{2}}{4}+\omega_{\mathrm{pe}}^{2}\right)^{1 / 2}$,

the extraordinary mode does not propagate at any angle $\theta$. The region between the reflection point and the cold plasma resonance for the angle $\theta$ [Eq. (32)] is evanescenl.

If the region of evanescence is sufficiently thin (due to low density or short magnetic scale length) a full wave treatment shows that energy incident from the cutoff side can tunnel through the evanescent region to be absorbed at the resonance or transmitted.13,20 in particular, for waves propagating along the magnetic field In a parallel stratified plasma the refractive index is given by Eq. (14) and the wave equation can be written in the form

$\frac{d^{2} E}{d z^{2}}+k_{0}^{2}\left(1+\frac{z_{0}}{z}\right) E=0$ 
where

$$
\begin{aligned}
& k_{0}=\frac{\omega}{c}=\text { free space wave number, and } \\
& z_{0}=\frac{\omega_{\text {pe }}^{2}}{\omega^{2}} L=\text { thickness of the cutoff region. }
\end{aligned}
$$

We have assumed a linear magnetic field profile $\Omega=\omega(1+z / L)$ and constant density. The Budden analysis for waves propagating from the cutoff side gives a power reflection coefficient $R$, a power transmission coefficient $T$, and an absorption coefficient $A$ of the form

$$
\begin{aligned}
& R=\left(1-e^{-\pi k_{0} z_{0}}\right)^{2}, \\
& T=e^{-\pi k_{0} z_{0}}, \\
& A=1-R-T=e^{-\pi k_{0} z_{0}}\left(1-e^{-\pi k_{0} z_{0}}\right) .
\end{aligned}
$$

If the cutoff zone is greater than one free space wavelength thick $\left(k_{0} z_{0}>2 \pi\right)$, the reflection coefficient is essentially unity $\{R=[1-$ $\left.\left.\exp \left(-2 \pi^{2}\right)\right]^{2} \cong 1-5 \times 10^{-9}\right\}$. For very small $k_{0} z_{0}$ the wave is completely transmitted. The absorption coefficient has a maximum $A=1 / 4$ for $z_{0}$ such that $\exp \left(-\pi k_{0} z_{0}\right)=1 / 2$. From Fig. 15 it is clear that the central region of EBT is completely cut off from the extraordinary mode. However, in the low density region near the coil casing the cutoff zone is thin $\left(z_{0} \cong 0.25 \mathrm{~cm}\right.$ ). This value gives $R=0.88, \mathrm{~L}=0.06$, and $\mathrm{A}=0.06$. Therefore, it is likely that in this low density surface region, extraordinary mode energy can be weakly absorbed and transmitted into the high field region.

The picture which is emerging is that microwave energy injected as extraordinary mode near the midplane is essentially trapped by the right-hand cutoff and undergoes many reflections at the cutoff and cavity wall until it is absorbed or transmitted by tunneling or until it is converted to ordinary mode. Similarly, since the ordinary mode is so very weakly damped in EBT-I, the principal sink for this form of radiation 
is conversion to extraordinary mode. These considerations suggest that conversion between ordinary mode and extraordinary mode may play an important role in the microwave energy balance in EBT. An investigation is under way of the various mechanisms for such mode conversion, particularly depolarization upon reflection by a metallic wall.

It should be emphasized that the proper treatment of singular surfaces is critical if meaningful results are to be obtained from the study. The one incontrovertible piece of experimental evidence is that. heating does occur in EBT; therefore, any theory which seeks to explain microwave heating in EBT must have provision for the microwaves to tunnel through, mode convert past, or otherwise get around the righthand cutoff. 


\section{NONLINEAR PROCESSES}

EBT is powered by a more or less coherent, large amplitude $\mathrm{rf}$ wave. A concern was that this large amplitude wave might drive nonlinear processes which would invalidate the linear wave propagation and damping model. In particular, cavitation due to the ponderamotive force and parametric wave decay processes were considered. It turns out that for EBT the ponderamotive potential is quite small in comparison to the thermal energy and is therefore unlikely to effect the plasma density significantly. With respect to parametric processes, there are two principal questions:

(1) To what extent do parametric processes deplete the wave energy before propagation of the microwaves to the linear resonance zones?

(2) What role might damping of parametric decay products play in plasma heating?

The elementary calculations discussed here suggest that thresholds for most parametric decay processes are too high to occur in EBT. However, this conclusion must be considered tentative since only a few of the multitude of possible decay combinations have been considered.

Whether or not any nonlinear process occurs depends on the magnitude of the microwave electric field. The microwave electric field strength can be estimated by assuming that the total wave energy density $\mathrm{U}_{\mathrm{T}}$ is uniform throughout the cavity volume. Then using the relation between the observed cavity $Q \approx 10$ and the injected microwave power $P_{\mu} \cong 60 \mathrm{~kW}$,

$Q=\frac{\int d^{3} x U_{T}}{P_{\mu}} f_{\mu}$,

one obtains for the wave energy density of the 18-GHz microwaves

$$
\mathrm{U}_{\mathrm{T}}=\frac{\mathrm{QP}}{\mathrm{vf_{ \mu }}}=2.5 \times 10^{-11} \mathrm{~J} / \mathrm{cm}^{3},
$$


where $\mathrm{f}_{\mu}=$ microwave frequency and $\mathrm{v}=1.35 \times 10^{6} \mathrm{~cm}^{3}$ is the cavity volume. Since $U_{T}=U_{E M}+U_{p l a s m a}$ where $U_{E M}=\varepsilon_{0} E^{2}$ and $U_{p l a s m a}>0$ for a stable plasma, the electric field is bounded by $E \leqslant 16.7 \mathrm{v} / \mathrm{cm}$. of course, WKB amplification of the wave fields can occur near cutoffs and resonances 20 but except for very special circumstances, this amplification is less than an order of magnitude.

To obtain an indication of the importance of parametric decay processes in EBT, the particular case of decay of an upper hybrid wave into an upper hybrid wave and an unmagnetized ion acoustic wave was considered. The upper hybrid mode seems particularly appropriate for the pump wave since an upper hybrid resonance layer extends across the plasma and resonant wave focusing may occur there. Other low frequency modes were considered briefly (lower hybrid and inn cyclotron modes), but it was not possible to find $k_{1}, k_{\|}$values which were compatible with the upper hybrid mode and still solvable analytically.

The threshold condition for this decay process can be written in the dipole approximation, 21,22

$\frac{E_{T}^{2}}{8 \pi n_{e} l^{\prime}} \cong 8 \frac{\omega_{2}^{2}}{\omega_{p e}^{2}} \frac{\Gamma_{1}}{\omega_{1}} \frac{\Gamma_{2}}{\omega_{2}}$

where

$\mathrm{E}_{\mathrm{T}}=$ threshold pump wave field,

$\omega \omega_{2}^{2}=\omega_{\mathrm{pe}}^{2}+\Omega_{\mathrm{e}}^{2} \cong \omega_{\mu}^{2}$ is frequency of the high frequency mode, $\Gamma_{2} \cong \frac{\sqrt{k}}{k^{2} \lambda_{D e}^{2}} \frac{\omega_{\text {pe }}^{2}}{2 k_{\|} v_{e}} \exp \left[-\frac{\left(\omega_{2}-s_{e}\right)^{2}}{4 k_{\|}^{2} v_{e}^{2}}\right]=$ damping rate of high frequency mode

$\omega_{1}^{2}=\mathrm{k}^{2} \omega_{\mathrm{pi}}^{2} \lambda_{\mathrm{De}}^{2}=$ frequency of low frequency mode, and $\Gamma_{1}=\omega_{1} \sqrt{\frac{\pi}{2^{3 / 2}}}\left(\frac{k}{k_{y}} \sqrt{\frac{\mathrm{m}_{e}}{\mathrm{~m}_{i}}}+\frac{\mathrm{T}_{\mathrm{e}}}{\mathrm{T}_{i}} e^{-\mathrm{T}_{\mathrm{e}} / 2 \mathrm{~T}_{i}}\right)=$ damping rate of low frequency mode. 
Parameters characteristic of the edge of an EBT-I plasma were assumed:

$$
\begin{aligned}
\mathrm{T}_{\mathrm{e}} & =200 \mathrm{eV}, \\
\mathrm{T}_{i} & =50 \mathrm{eV}, \\
\mathrm{f}_{\mu} & =\omega_{\mu} / 2 \mathrm{k}=20 \mathrm{GHz}, \\
\omega_{\mu}^{2} & =\Omega_{\mathrm{e}}^{2}+\omega_{\mathrm{pe}}^{2}, \\
\omega_{\mathrm{pe}} & =\Omega_{\mathrm{e}} / 2 \rightarrow \mathrm{n}_{\mathrm{e}} \cong 10^{12} .
\end{aligned}
$$

Because of the curvature and gradients along the magnetic field, the parallel wavelength is limited such that $\lambda_{\|} \ll \mathrm{L}_{\|}$where $\mathrm{L}_{\|}$is a scale. length for plasma variations along the field lines. For EBT an upper limit for $\lambda_{\|}$would appear to be about $2 \mathrm{~cm}$; therefore, $k_{\|}(\min ) \geq$ $2 \pi / 2 \mathrm{~cm}$. The minimum damping for the upper hybrid mode is obtained by setting $k_{\|}=k_{\|(\min )}=\pi / \mathrm{cm}$. With these assumptions, the threshold condition becomes

$\frac{E_{T}^{2}}{8 \pi n_{e}^{T} e} \cong 5 \times 10^{-4}$

or $E_{0} \gtrsim 700 \mathrm{v} / \mathrm{cm}$ for instability. This is of course much higher than the microwave field strengths to be expected unless considerable amplification occurs at the upper hybrid layer.

Namkung 23 has recently done a thorough $\left(k_{\|}, k_{\perp}\right)$ space study of the decay of an upper hybrid wave to an upper hybrid wave and an ion acoustic wave. He has found cases in which the primary damping mechanism is collisional and which have threshold fields in the range $\mathrm{E}_{\mathrm{T}} \sim 30-100 \mathrm{~V} / \mathrm{cm}$. These values are still larger than the field strengths which could be expected to occur in EBT. In addition, decay thresholds can be raised considerably by effects of plasma inhomogeneity, finite pump extent, and pump depletion.

In view of the rather high threshold fields found in these calculations, at present parametric instabilities appear unlikely to play a major role in EBT devices. The number of parametric decay combinations is quite large and a more thorough search should be performed particularly for decay to ion quasi-modes. However, this work must have a somewhat lower priority than other aspects of the microwave heating study. 


\section{CONCLUSIONS}

The microwave heating study for EBT can be divided into four areas which are to some extent independent of one another: (1) geometrical optics, (2) heating mechanisms, (3) connection formulae across regions of geometrical optics breakdown, and (4) nonlinear effects. A basic component of the study is the development of the geometrical optics code, RAYS. The code is now capable of calculating ray trajectories, wave number, and electric field polarization in three-dimensional, cold plasma equilibria. A finite temperature wave damping model has been included to calculate energy deposition. The most pressing needs for the RAYS code are: inclusion of finite temperature effects on ray paths, inclusion of mode conversion and reflection at çonducting wall.s, and modeling of the injected microwave fields near the antenna.

The heating process can be understood on the basis of single particle orbits in specified microwave fields, or on the basis of linear wave damping. Energy deposition calculations including self-consistent wave fields and quasi-linear effects have not yet been done. Extensive coding will be required in the RAYS code in order to keep track of the spatial distribution of energy deposition. Absorption at the second harmonic resonant zone also needs to be included in the RAYS code.

Work on developing the joining relations across sutoffs and resonances and the task of linear mode conversion problems have barely begun. This is the area which will require the most analysis before the RAYS code can be used to calculate complicated energy deposition process.

Elementary calculations have been performed to evaluate the importance of ponderamotive force and parametric decay processes on wave propagation in EBT. The results are tentative but indicate that these nonlinear processes are unlikely to play a major role. More work needs to be done but this must be given a lower priority.

A complete investigation of the electron cyclotron resonance heating in EBT, including wall effects, linear mode conversion, tunneling, etc., must still be considered a long-term project. However, with a moderate amount of additional analysis and coding, problems which do not involve such complications could be handled in the reasonably near future. 
Situations which could be treated are the heating in EBT devices or tokamaks due to the ordinary mode and heating in tokamaks due to the extraordinary mode, provided injection is from the inside and avoids the right-hand cutoff. 


\section{REFERENCES}

1. R. A. Dandl et al., Proc. 5th Conf. on Plasma Physics and controlled Nuclear Fusion Research, Vol. II, p. 141 (1975); R. A. Dandl et al., Research Program for Plasma Confinement and Heating in ELMO Bumpy Torus Devices, ORNL/TM-4941, Oak Ridge, Tennessee (June 1975); R. A. Dandl et al., The ELMO Bumpy Toms Program, ORNL/TM-5451, Oak Ridge, Tennessee (April 1976).

2. R. A. Dand 1, H. O. Eason, and H. Ikegami, "Microwave Plasma Heating at the Electron Cyclotron Frequency," to be published in Nucl. Fusion.

3. V. E. Golant ct al., Sov. Phys.-Tcch, Phys. 17, 488 (1972); V. V, Alikaev et al., Nucl. Fusion Supplement Plasma Physics and Controlzed Nuclear Fusion Research, p. 33 (1974).

4. H. Grawe, P1asma Phys. 11, 151 (1969).

5. 0. C. Eldridge, Phys. Fluids 15, 676 (1972).

6. M. Brambilla, Plasma Phys. 10, 359 (1968).

7. J. C. Sprott, Phys. Fluids 14, 1795 (1971).

8. V. V. Alikaev, Perspectives on Utilization of Electron-Cyclotron Waves for Heating Plasmas in Large-scale Devices of the Type "Tokamak," IAE-2610, Moscow (1976).

9. A. G. Litvak et al., Nucl. Fusion 17, 659 (1977).

10. 0. C. Eldridge, W. Namkung, and A. C. England, Electron Cyclotron Heating in Tokamaks, ORNL/TM-6052, Oak Ridge, Tennessee (November 1977), to be published in Phys. Fluids.

11. A. F. Kukes, Plasma Phys. 10, 367 (1968).

12. E. F. Jaeger, D. A. Spong, and C. L. Hedrick, Phys, Rev. Lett. 40, 866 (1978); E. F. Jaeger and C. L. Hedrick, A Numerical Model for Radial Transport in the ELMO Bumpy Torus, Oak Ridge, Tennessee (November 1977).

13. T. H. Stix, The Theory of PZasma Waves, McGraw-Hill, New York, 1962.

14. A. I. Akhiezer et al., Plasma Electrodynamics, Vo1. I, Pergamon Press, Oxford, 1975.

15. Ira B. Bernstein, Phys. Fluids 18 , 320 (1975). 
16. E. F. Jaeger, A. J. Lichtenberg, and M. A. Lieberman, Plasma Phys. 14, 1073 (1972); M. A. Lieberman and A. J. Lichtenberg, Plasma Phys. 15, 125 (1973).

17. E. F. Jaeger (Oak Ridge National Laboratory), private communication 1977.

18. K. G. Budden, Radio Waves in the Ionosphere, Cambridge University Press, Cambridge, 1961.

19. G. A. Kriegsman, J. Math. Phys. 17, 112 (1976).

20. R. B. White and F. F. Chen, Plasma Phys. 16, 565 (1974).

21. P. K. Kaw, p. 179 in Advances in Plasma Physics, ed. by Albert Simon and William B. Thompson, John Wiley and Sons, New York, 1976.

22. M. Porkolab, Proc. 12th Int. Conf. on Phenomena in Ionized Gases, Part 2 (Invited Papers), p. (1975).

23. Won Namkung, Ph.D. thesis, University of Tennessee, Knoxville, Tennessee (1977). 
THIS PAGE

\section{WAS INTENTIONALLY LEFT BLANK}


ORNL/TM-6320

Dist. Category UC-20 g

INTERNAL DISTRIBUTION

1. L. A. Berry

2. J. D. Callen

3. R. A. Dand 1

4. R. A. Dory

5. G. G. Kelley

6. H. H. Haselton

7. P. N. Haubenreich

8. M. S. Lube11

9. 0. B. Morgan

10. H. Postma

11. M. W. Rosentha1
12. J. Sheffield

13. D. Steiner

14-47. D. B. Batchelor

48-49. Laboratory Records Department

50. Laboratory Records, ORNL-RC

51. Document Reference Section

52-53. Central Research Library

54. Fusion Energy Division Library

55. Fusion Energy Division

Communications Center

56. ORNL Patent Office

\section{EXTERNAL DISTRIBUTION}

57. D. J. Anthony, Energy Systems and Technology Division, General Electric Company, 1 River Road, Bldg. 23, Room 290, Schenectady, NY 12345

58. Bibliothek, Max-Planck Institute für Plasmaphysik, 8046 Garching bei München, Federal Republic of Germany

59. Bibliothèque, Service du Confinement des Plasmas, C.E.A., B.P. No. 6, 92, Fontenay-aux Roses (Seine), France

60. Lung Cheung, Department of Electronics, University Science Center, The Chinese University of Hong Kong, Shatin, N.T., Hong Kong

61. J. F. Clarke, Office of Fusion Energy, G-234, Department of Energy, Washington, DC 20545

62. R. W. Conn, Fusion Technology Program, Nuclear Engineering Department, University of Wisconsin, Madison, WI 53706

63. CTR Library, c/o Alan F. Haught, United Technologies Research Laboratory, East Hartford, CT 06108

64. CTR Reading Room, c/o Allan N. Kaufman, Physics Department, University of California, Berkeley, CA 94720

65. J. Nar1 Davidson, School of Nuclear Engineering, Georgia Inst1tute of Technology, Atlanta, GA 30332

66. Documentation S.I.G.N., Départment de la Physique du Plasma et de la Fusion Controlée, Association EURATOM-CEA sur la Fusion, Centre d'Etudes Nucléaires, B.P. 85, Centre du TRI, 38041 Grenoble, Cedex, France

67. W. R. Ellis, Office of Fusion Energy, G-234, Department of Energy, Washington, DC 20545

68. Harold K. Forsen, Exxon Nuclear Co., Inc., 777 106th Avenue, N.E., C-000777, Bellevue, WA 98009

69. Harold P. Furth, Princeton Plasma Physics Laboratory, Princeton University, Forrestal Campus, P.0. Box 451, Princeton, NJ 08540

70. Roy W. Gould, California Institute of Technology, Mail Stop 116-81, Pasadena, CA 91125 
71. Robert. L. Hirsch, Exxon Research and Engineering, P.0. Box 101, .Florham Park, NJ 07932

72. Kaymond A. Huse, Manager, Research and Development, Public Service Gas and Electric Company, 80 Park Place, Newark, NJ 07101

73. T. Hsu, Office of Fusion Energy, G-234, Department of Energy, Washington, DC 20545

74. V. E. Ivanov, Physical-Technical Institute of the Ukranian Academy of Sciences, Sukhumi, U.S.S.R.

75. A. Kadish, Office of Fusion Energy, G-234, Department of Energy, Washington, DC 20545

76. L. M. Kovrizhnikh, Lebedev Institute of Physics, Academy of Sciences of the U.S.S.R., Leninsky Prospect 53, Moscow, U.S.S.R.

77. Guy Lava1, Groupe de Physique Thèorique, Ecole Polytechnique, 91 Palaiseau, Paris, France

78. Library, Centre de Recherches en Physique des Plasma, 21 Avenue des Bains, 1007, Lausanne, Switzerland

79. Library, Culham Laboratory, United Kingdom Atomic Energy Authority, Abingdon, Oxon, 0x14 3DB, United Kingdom

80. Library, FOM-Institut voor Plasma - Fysica, Rijnhuizen, Jutphaas, Netherlands

81. Library, Inst1tute for P1asma Physics, Nagoya University, Nagoya, Japan 464

82. Library, International Centre for Theoretical Physics, Trieste, Italy

83. Library, Laboratorio Gas Ionizzati, Frascati, Italy

84. Dsumber G. Lominadze, Academy of Sciences of the Georgian S.S.R., 8 Dzerzh1nsk1 St., 38004, Tbilisi, U.S.S.R.

85. Oscar P. Manley, Office of Fusion Energy, G-234, Department of Energy, Washington, DC 20545

86. D. G. McAlees, Exxon Nuclear Co., Inc., Research and Technology Laser Enrichment Department, 2955 George Washington Way, Richland, WA 99352

87. J. E. McCune, School of Engineering, Department of Aeronautics and Astronautics, Bldg. 37-391, Massachusetts Institute of Technology, Cambridge, MA 02139

88. Claude Mercier, Service du Theorie des Plasmas, Centre d'Études Nucléaires, Fontenay-aux-Roses (Seine), France

89. K. G. Moses, Office of Fusion Energy, G-234, Department of Energy, Washington, DC 20545

90. D. Pfirsch, Institute for Plasma Physics, 8046 Garching bei Muinchen, Federal Republic of Germany

91. Plasma Physics Group, Department of Engineering Physics, Australian National University, P.0. Box 4, Canberra A.C.T. 2600, Australia

92. A. Rogister, Institute for Plasma Physics, KFA, Postfach 1913, D-5170, Jiilich 1, Federa1 Republic of Germany

93. W. Sadowski, Office of Fusion Energy, G-234, Department of Energy, Washington, DC 20545

94. V. D. Shafranov, I. V. Kurchatov Institute of Atomic Energy, 46 Ulitsa Kurchatova, P.0. Box 3402, Moscow, U.S.S.R. 
95. Yu. S. Sigov, Institute of Applied Mathematics of the U.S.S.R. Academy of Sciences, Miuskaya, Sq. 4, Moscow A-47, U.S.S.R.

96. W. M. Stacey, Jr., School of Nuclear Engineering, Georgia Institute of Technology, Atlanta, GA 30332

97. J. B. Taylor, Culham Laboratory, United Kingdom Atomic Energy Authority, Abingdon, Oxon, OX14 3DB, United Kingdom

98. Thermonuclear Library, Japan Atomic Energy Research Institute, Toka1, Naka, Ibaraki, Japan

99. Francisco Verdaguer, Director, Division of Fusion, Junta de Energia Nuclear, Madrid 3, Spain

100. Director, Research and Technical Support Division, Department of Energy, Oak Ridge Operations, P.O. Box E, Oak Ridge, TN 37830

101-276. Given distribution as shown in TID-4500, Magnetic Fusion Energy (Distribution Category UC-20 g, Theoretical Plasma Physics) 